OPEN ACCESS

Edited by: John D. Wade

University of Melbourne, Australia

Reviewed by:

Mario Zoratti,

National Research Council (CNR), Italy Elizabeth New,

The University of Sydney, Australia

${ }^{*}$ Correspondence:

Michael P. Murphy

mpm@mrc-mbu.cam.ac.uk

Richard C. Hartley

richard.hartley@glasgow.ac.uk

Specialty section:

This article was submitted to

Chemical Biology

a section of the journal

Frontiers in Chemistry

Received: 27 April 2020

Accepted: 27 July 2020

Published: 09 September 2020

Citation:

Pala L, Senn HM, Caldwell ST, Prime TA, Warrington S, Bright TP, Prag HA, Wilson C, Murphy MP and

Hartley RC (2020) Enhancing the Mitochondrial Uptake of Phosphonium

Cations by Carboxylic Acid

Incorporation. Front. Chem. 8:783.

doi: 10.3389/fchem.2020.00783

\title{
Enhancing the Mitochondrial Uptake of Phosphonium Cations by Carboxylic Acid Incorporation
}

\author{
Laura Pala ${ }^{1}$, Hans M. Senn ${ }^{1}$, Stuart T. Caldwell ${ }^{1}$, Tracy A. Prime ${ }^{2}$, Stefan Warrington ${ }^{1}$, \\ Thomas P. Bright ${ }^{2}$, Hiran A. Prag ${ }^{2}$, Claire Wilson ${ }^{1}$, Michael P. Murphy ${ }^{2,3 *}$ and \\ Richard C. Hartley ${ }^{1 *}$ \\ ${ }^{1}$ School of Chemistry, University of Glasgow, Glasgow, United Kingdom, ${ }^{2}$ MRC Mitochondrial Biology Unit, University of \\ Cambridge, Cambridge, United Kingdom, ${ }^{3}$ Department of Medicine, University of Cambridge, Cambridge, United Kingdom
}

There is considerable interest in developing drugs and probes targeted to mitochondria in order to understand and treat the many pathologies associated with mitochondrial dysfunction. The large membrane potential, negative inside, across the mitochondrial inner membrane enables delivery of molecules conjugated to lipophilic phosphonium cations to the organelle. Due to their combination of charge and hydrophobicity, quaternary triarylphosphonium cations rapidly cross biological membranes without the requirement for a carrier. Their extent of uptake is determined by the magnitude of the mitochondrial membrane potential, as described by the Nernst equation. To further enhance this uptake here we explored whether incorporation of a carboxylic acid into a quaternary triarylphosphonium cation would enhance its mitochondrial uptake in response to both the membrane potential and the mitochondrial $\mathrm{pH}$ gradient (alkaline inside). Accumulation of arylpropionic acid derivatives depended on both the membrane potential and the $\mathrm{pH}$ gradient. However, acetic or benzoic derivatives did not accumulate, due to their lowered $\mathrm{pK}_{\mathrm{a}}$. Surprisingly, despite not being taken up by mitochondria, the phenylacetic or phenylbenzoic derivatives were not retained within mitochondria when generated within the mitochondrial matrix by hydrolysis of their cognate esters. Computational studies, supported by crystallography, showed that these molecules passed through the hydrophobic core of mitochondrial inner membrane as a neutral dimer. This finding extends our understanding of the mechanisms of membrane permeation of lipophilic cations and suggests future strategies to enhance drug and probe delivery to mitochondria.

Keywords: mitochondria, phosphonium, mitochondria-targeting, membrane permeation, membrane potential, pH gradient, computational chemistry

\section{INTRODUCTION}

Mitochondrial dysfunction contributes to a wide range of pathologies, consequently they are an important therapeutic target (Nunnari and Suomalainen, 2012; Gorman et al., 2016; Murphy and Hartley, 2018). Furthermore, the delivery of probe molecules to the organelle in vivo is essential in understanding how mitochondrial dysfunction arises (Yousif et al., 2009; Smith et al., 2012; Logan et al., 2014; Jean et al., 2016). There are a number of strategies to deliver molecules to 
mitochondria in vivo, with conjugation to the alkyltriphenylphosphonium (TPP) cation being the most widespread (Smith et al., 2003, 2012; Yousif et al., 2009). TPP is used because it readily crosses biological membranes without the requirement for a protein carrier, and is chemically tractable making it easy to introduce synthetically into the molecule to be targeted (Smith et al., 2011, 2012), as well as having a good safety and toxicity profile. An important aspect of the TPP targeting system is that its positive charge and the large mitochondrial membrane potential lead to these molecules accumulating $\sim 1,000$-fold within energized mitochondria, as described by the Nernst equation (Ross et al., 2006; Smith et al., 2011). Even so, improving mitochondria-targeting head groups to enhance mitochondrial accumulation would lead to improved therapies and probes.

To develop an improved mitochondria-targeting head group, we started from the finding that a TPP cation conjugated to a long alkyl chain carboxylic acid 1 accumulates within mitochondria to a greater extent than related compounds without an acid moiety (Figure 1) (Finichiu et al., 2013). This greater uptake occurs because of the equilibrium between the protonated (1) and deprotonated forms (2) determined by the $\mathrm{pK}_{\mathrm{a}}$ of the carboxylic acid ( 4.9) (Kanicky and Shah, 2003). The higher $\mathrm{pH}$ of the mitochondrial matrix $(\sim 8)$ relative to the cytosol $(\sim 7.2)$ and the inability of the zwitterionic TPP carboxylate 2 to cross the mitochondrial inner membrane means that the overall accumulation of the molecule is enhanced 4-10-fold compared to a comparable TPP without a carboxylic acid (Finichiu et al., 2013). Hence, we set out to develop a generic TPP-carboxylic acid targeting group so as to enhance mitochondrial uptake 410-fold over current TPP groups (Figure 1). The simplest way to do this is to incorporate a carboxylic acid group on one of the TPP phenyls. Both protonated and deprotonated forms of the carboxylic acid would be present in a ratio depending on the $\mathrm{pK}_{\mathrm{a}}$ of the acid and the local $\mathrm{pH}$ (Figure 1). Only the protonated form of the TPP-carboxylic acid targeting group should cross the mitochondrial membrane and once within the matrix, the higher $\mathrm{pH}$ should increase accumulation of the membrane-impermeant deprotonated form. Thus, this approach should generate a generic TPP-carboxylic acid targeting group to increase the delivery of the same cargo targeted with a TPP group (Figure 1). Furthermore, this combined TPP-carboxylic acid targeting group could easily be attached to any cargo using a simple phosphine-carboxylic acid precursor. Here we set out to develop such a generic TPP-carboxylic acid targeting group to enhance the delivery of drugs and probes to mitochondria in vivo.

\section{MATERIALS AND METHODS}

\section{Synthetic Chemistry}

The test compounds 3-19 were prepared as shown in Scheme 1. Phosphines 20 and $\mathbf{2 1}$ were alkylated with iodomethane, 1bromohexane, or 1-bromododecane to give the corresponding salts 3-8 (Scheme 1). Carboxylic acids 3, 4, 7 were converted into the corresponding ethyl esters 12, 13, and 15 by stirring in ethanol with catalytic concentrated sulfuric acid. The trifluoroethyl ester 19 was prepared from carboxylic acid 7 in a similar way using trifluoroethanol as solvent. However, a better approach to trifluoroethyl esters was to esterify the carboxylic acid group of the parent phosphines $\mathbf{2 0}$ and 21 with trifluoroethanol using diisopropylcarbodiimide (DIC) coupling with dimethylaminopyridine (DMAP) as a nucleophilic catalyst to give the corresponding esters $\mathbf{2 3}$ and $\mathbf{2 4}$, and then alkylate the phosphine to give the phosphonium salts 16-18. Ethyl ester 14 was prepared in a similar way using dicyclohexylcarbodiimide (DCC) coupling to make ester $\mathbf{2 2}$ followed by alkylation with iodomethane. The synthesis of the phenylpropionic acid derivatives 9-11 began with the preparation of iodoarene 25 by the method of (Qin et al., 2015) and protection of the acid as ester 26 (Scheme 2). Adapting the procedure reported by (Dydio et al., 2014), which we had used to prepare phosphine $\mathbf{2 1}$, iodoarene $\mathbf{2 6}$ was cross-coupled with diphenylphosphine and

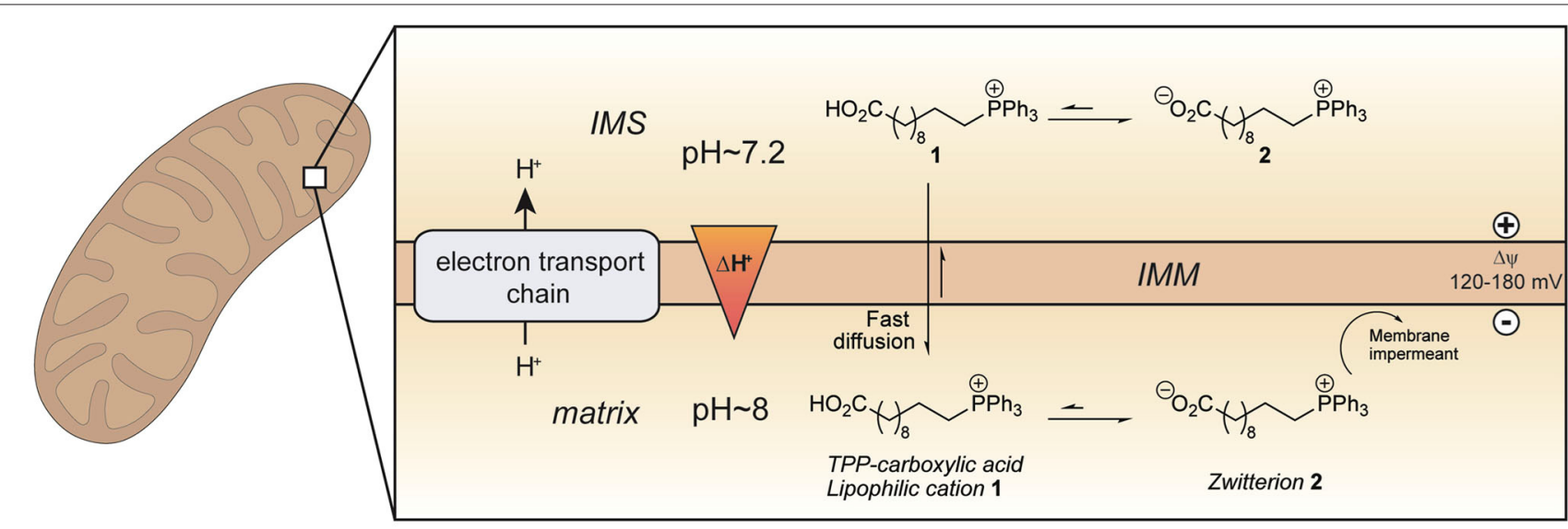

FIGURE 1 | Enhanced mitochondrial uptake by a TPP cation linked to a carboxylic acid. Model showing how a TPP long chain carboxylic acid $\mathbf{1}$ crosses the mitochondrial inner membrane and accumulates within the mitochondrial matrix in its zwitterionic form $\mathbf{2}$ due to the higher $\mathrm{pH}(\sim 8)$ in mitochondria compared to that in the cytosol ( 7.2). IMM, inner mitochondrial membrane; IMS, intermembrane space. 


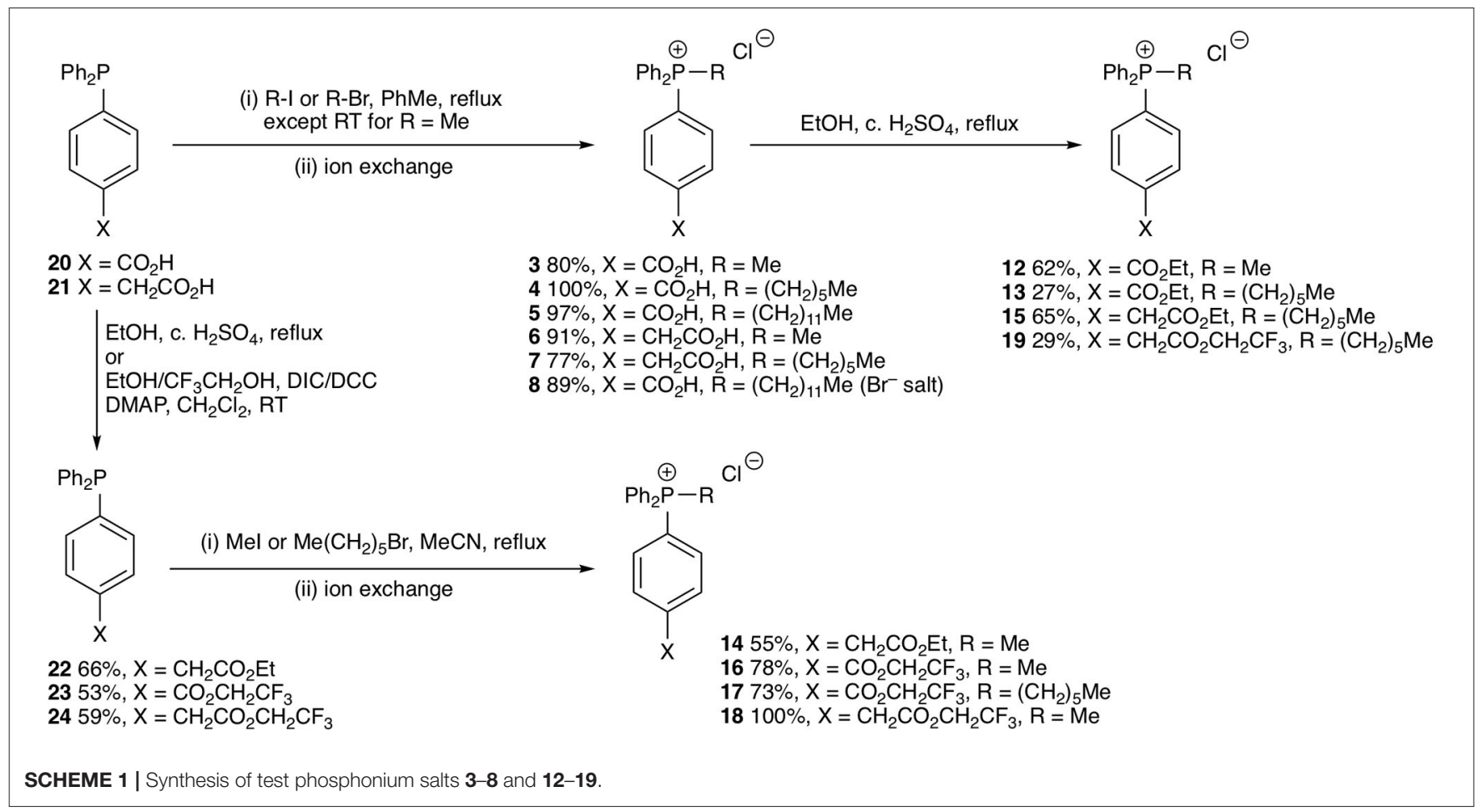

then alkylated in situ with iodomethane, 1-bromobutane, or 1bromohexane to give the corresponding phosphonium salts 2729. Acid-catalyzed hydrolysis then gave the phosphonium salts 9-11. The details of the synthetic procedures are described in the Supplemental Information.

\section{Biology-Methods}

\section{Animals}

All procedures were carried out in accordance with the UK Animals (Scientific Procedures) Act 1986 and the University of Cambridge Animal Welfare Policy. Female Wistar rats (typically 6-10 weeks old, weighing $\sim 200$ g, Charles River Laboratories, UK) were housed in pathogen-free facilities at $21 \pm 2^{\circ} \mathrm{C}$, humidity $57 \pm 5 \%$ with a $12 \mathrm{~h}$ light/dark cycle and laboratory chow and water were available ad libitum.

\section{Isolation of Rat Liver and Heart Mitochondria}

Rats were killed by stunning and cervical dislocation and the liver or heart rapidly excised and transferred to ice cold mitochondrial isolation buffer. Mitochondria were isolated from rat liver tissue by differential centrifugation in STE buffer [250 mM sucrose, $5 \mathrm{mM}$ Tris, $1 \mathrm{mM}$ ethyleneglycol-bis( $\beta$-aminoethylether)- $N, N$, $N^{\prime}, N^{\prime}$-tetraacetic acid (EGTA) (pH 7.4 at $4^{\circ} \mathrm{C}$, adjusted with $\mathrm{HCl})$ ] (Chappell and Hansford, 1972). The liver was washed in ice-cold STE, chopped finely, rinsed in STE and homogenized 5-8 times using a loose PTFE pestle in a $55 \mathrm{ml}$ Potter-Elvehjem tissue grinder (Wheaton, USA) and then with a tight pestle. The homogenate was centrifuged $\left(1,000 \times \mathrm{g}, 3 \mathrm{~min}, 4^{\circ} \mathrm{C}\right)$, the pellets were discarded, and the supernatants centrifuged $(10,000 \mathrm{x} \mathrm{g}$, $\left.10 \mathrm{~min}, 4^{\circ} \mathrm{C}\right)$. The supernatant was discarded and the pellet was resuspended and centrifuged again $\left(10,000 \times \mathrm{g}, 10 \mathrm{~min}, 4^{\circ} \mathrm{C}\right)$.

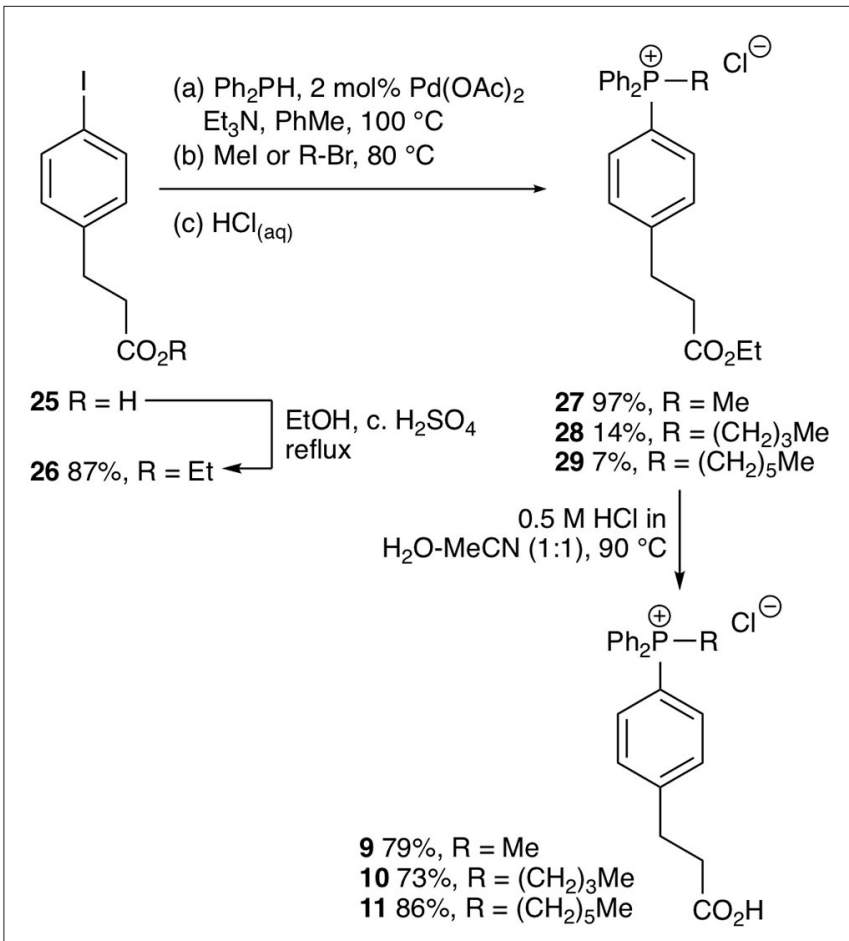

SCHEME 2 | Synthesis of test phosphonium salts 9-11.

The final mitochondria pellet was resuspended in $\sim 5 \mathrm{~mL}$ STE buffer, kept in ice and used within $4 \mathrm{~h}$.

Rat hearts were washed in ice-cold STEB buffer [STE buffer supplemented with $0.1 \%$ (ws/v) fatty-acid free bovine 
serum albumin (BSA)], chopped finely, washed again and then homogenized 5-8 times using a loose PTFE pestle in a $55 \mathrm{~mL}$ Potter-Elvehjem tissue grinder (Wheaton, USA) and then a tight pestle with roughly 8 strokes. The homogenate was centrifuged $\left(700 \times \mathrm{g}, 5 \mathrm{~min}, 4^{\circ} \mathrm{C}\right)$ and the supernatant was decanted through two layers of a pre-wetted muslin into new centrifuge tubes. The pellet was resuspended and centrifuged again $(700 \times \mathrm{g}$, 5 min, $4^{\circ} \mathrm{C}$ ). This supernatant was also decanted through muslin and the combined supernatants centrifuged $(10,000 \times \mathrm{g}, 10 \mathrm{~min}$, $\left.4{ }^{\circ} \mathrm{C}\right)$. The mitochondrial pellet was resuspended in STEB and centrifuged again $\left(10,000 \times \mathrm{g}, 10 \mathrm{~min}, 4^{\circ} \mathrm{C}\right)$. The mitochondrial pellet was resuspended in STE without BSA ( $\sim 300 \mu \mathrm{L}$ per heart), kept on ice and used within $4 \mathrm{~h}$. The protein concentration of the mitochondrial suspensions was determined using the BCA assay using BSA as a standard (Thermo Fisher Scientific, UK).

\section{Reversed Phase-High Performance Liquid Chromatography (RP-HPLC)}

For most RP-HPLC experiments, the stationary phase used was a C18 column (Jupiter $300 \AA$ A, Phenomenex) with a Widepore C18 guard column (Phenomenex). The column was connected to a 321 pump, a UV/Vis 151 system (Gilson, UK), a RIC20 Remote Controlled Chilling/Heating Dry bath (Torrey Pine Scientific) and a GX-241 liquid handler (Gilson) with Trilution LC 3.0 software (Gilson). The mobile phase was a gradient of water $+0.1 \%(\mathrm{v} / \mathrm{v})$ trifluoroacetic acid (TFA) (HPLC buffer A) and acetonitrile [HPLC grade acetonitrile (Fisher Scientific, UK)] + $0.1 \%(\mathrm{v} / \mathrm{v})$ TFA (HPLC buffer B) at a flow rate of $1 \mathrm{~mL} / \mathrm{min}$. Samples were prepared in $1 \mathrm{~mL}$, containing $25 \%$ acetonitrile $+0.1 \%(\mathrm{v} / \mathrm{v})$ TFA. Prior to analysis, they were filtered under vacuum using Protein Precipitation Plates (Porvair, $100 \mu \mathrm{m}$ prefilter frit, $<10 \mu \mathrm{m}$ secondary frit). The filtrate was transferred to centrifuge tubes and placed in the chilled sample holder. 800 $\mu \mathrm{L}$ of sample were loaded into a $2 \mathrm{~mL}$ sample loop and injected onto the column for analysis. Compounds were eluted using the following gradient: 0-2 min: $5 \%$ buffer B, 2-17 min: 5-100\% buffer B, 17-19 min: 100\% buffer B, 19-22 min: 100-5\% buffer B. For some RP-HPLC experiments samples were prepared in $850 \mu \mathrm{L}$, containing $20 \%$ acetonitrile $+0.1 \%(\mathrm{v} / \mathrm{v})$ TFA. This was transferred to centrifuge tubes and placed in the chilled sample holder. $800 \mu \mathrm{L}$ of sample were loaded into a $2 \mathrm{~mL}$ sample loop and injected onto the column for analysis. Compounds were eluted using the following gradient: $0-2$ min: $5 \%$ buffer $\mathrm{B}, 2-4$ min: $55 \%$ buffer B, $4-16$ min: $70 \%$ buffer B, $16-18$ min: $70-100 \%$ buffer B, 18-21 min: 100\% buffer B, 21-23 min: 100-5\% buffer B. All samples were detected at $220 \mathrm{~nm}$ and peaks identified by comparing the retention times of the known standards.

\section{Mitochondrial Incubation of Compounds}

Rat liver or heart mitochondria $(0.5 \mathrm{mg}$ protein $/ \mathrm{mL})$ were incubated in $2 \mathrm{~mL} \mathrm{KCl}$ buffer $(120 \mathrm{mM} \mathrm{KCl}, 10 \mathrm{mM}$ HEPES, 1 mM EGTA, adjusted with $\mathrm{KOH}$ to $\mathrm{pH} 7.2,37^{\circ} \mathrm{C}$ ) supplemented with compound and internal standard $(5 \mu \mathrm{M}$ each, unless otherwise stated) in a shaking heat block $\left(37^{\circ} \mathrm{C}, 1,000 \mathrm{rpm}\right)$. Mitochondria were energized with succinate/rotenone (10 $\mathrm{mM} / 10 \mu \mathrm{M})$ or glutamate/malate $(10 \mathrm{mM}$ each). To abolish the mitochondrial proton motive force either FCCP $(1 \mu \mathrm{M})$, or a mixture of oligomycin, antimycin $\mathrm{A}$ and valinomycin (OAV) $(5 \mu \mathrm{g} / \mathrm{mL}, 100 \mathrm{nM}, 1 \mathrm{nM}$, respectively) were used. After the appropriate incubation time, the mitochondrial suspensions were rapidly cooled on ice and the mitochondria pelleted by centrifugation $\left(10,000 \times \mathrm{g}, 5 \mathrm{~min}, 4^{\circ} \mathrm{C}\right)$. The mitochondrial pellet was dried by aspirating the supernatant, snap frozen and stored at $-80^{\circ} \mathrm{C}$ until RP-HPLC analysis. For this, the pellet was thawed and extracted by resuspension in $250 \mu \mathrm{L}$ HPLC buffer B with vortexing, and then centrifuged $\left(16,000 \times \mathrm{g}, 10 \mathrm{~min}, 4^{\circ} \mathrm{C}\right)$. The resulting supernatant was removed and $750 \mu \mathrm{L}$ HPLC buffer A was added.

\section{Mitochondrial Respiration Assay}

Respiration of isolated mitochondria was assessed using the Oroboros Oxygraph-2K (O2K) high resolution respirometer (Oroboros Instruments, Austria), equipped with two stirred (200 rpm), and temperature controlled $\left(37^{\circ} \mathrm{C}\right)$ chambers. Before starting experiments, $2 \mathrm{~mL} \mathrm{KCl}$ buffer was left to equilibrate for approximately $1 \mathrm{~h}$ and the Oroboros was calibrated. Rat liver mitochondria $(2 \mathrm{mg}$ protein $/ \mathrm{mL}$ ) were then added to the chamber with glutamate and malate $(10 \mathrm{mM}$ each). The lid was closed, and respiration assessed. Compounds were added in increasing concentrations using gastight syringes (Hamilton Robotics, UK), with equal volumes of control compound being used in parallel experiments. Additions did not exceed $10 \mu \mathrm{L}$ per addition.

\section{QUANTUM-CHEMICAL CALCULATIONS}

\section{Computational Details}

Free energies of transfer and of ion-pairing/dimerisation were calculated using density-functional theory (DFT) with the TPSS exchange-correlation functional (Tao et al., 2003) (which is a pure meta-GGA functional) supplemented by Grimme's D3 correction (Grimme et al., 2010, 2011) to account for dispersive interactions. Structures and vibrational frequencies were calculated with the def2-SVP (cations) and def2-SVP+ (ion pairs and anions) basis sets, final energies with def2-TZVP+ in all cases (Weigend and Ahlrichs, 2005; Weigend, 2006). The "+" indicates augmentation of the published sets with one diffuse Gaussian function per valence angular momentum on all atoms; the exponents of the diffuse functions were derived such as to form a geometric series with the existing two outermost functions (even-tempered).

Structure optimisations and frequency calculations were performed with Turbomole 6.4 (Ahlrichs et al., 1989; Häser and Ahlrichs, 1989; Treutler and Ahlrichs, 1995; von Arnim and Ahlrichs, 1998, 1999), making use of the efficient MARI-J technique (Sierka et al., 2003). SCF energies were converged to $10^{-8} E_{\mathrm{h}}$; structures to $10^{-6} E_{\mathrm{h}}$ in the energy and $10^{-4} E_{\mathrm{h}} a_{0}^{-1}$ in the gradient. The COSMO continuum solvent model (Klamt and Schüürmann, 1993; Klamt and Jonas, 1996) was applied throughout, with default solvation radii and relative permittivity $\varepsilon_{\mathrm{r}}=78.3553$ and refractive index $n=1.3334$ for water and $\varepsilon_{\mathrm{r}}=1.8819, n=1.3749$ for hexane. Frequencies were obtained by numerical differentiation (NumForce), using only the "slow" component of solvent polarization (Klamt, 1996). All structures 
were confirmed by the absence of imaginary frequencies to be minima on the potential-energy surface in the respective solvent.

Final energies were calculated with Gaussian 09 (Frisch et al., 2013), using the SMD solvation model, which combines the IEFPCM polarisable continuum model for electrostatic solvation (Tomasi et al., 2005) with the SMD non-electrostatic terms (Marenich et al., 2009). The same values of $\varepsilon_{\mathrm{r}}$ and $n$ were used as above (which are G09 default values, noting that $n=\varepsilon_{\infty}{ }^{1 / 2}$ ). Default solvation radii were used, except for $\mathrm{Cl}$, whose solvation radius was reduced from $2.38 \AA$ to $1.8 \AA$ in order to reproduce better the experimental free energy of hydration (Hünenberger and Reif, 2011) of $\mathrm{Cl}^{-}, \Delta_{\text {solv }} G^{*}\left(\mathrm{Cl}^{-}\right.$, aq $)=-316.55 \mathrm{~kJ} \mathrm{~mol}^{-1}$; the default radius yielded $-271 \mathrm{~kJ} \mathrm{~mol}^{-1}$, the adjusted radius $-307 \mathrm{~kJ} \mathrm{~mol}^{-1}$. The solvation radius of $\mathrm{Na}$ was similarly reduced from $2.27 \AA$ to $1.6 \AA$; experimental $\Delta_{\text {solv }} G^{*}\left(\mathrm{Cl}^{-}\right.$, aq $)=-427.41$ $\mathrm{kJ} \mathrm{mol}^{-1}$, calculated using default radius: $-302 \mathrm{~kJ} \mathrm{~mol}^{-1}$, calculated using adjusted radius: $-429 \mathrm{~kJ} \mathrm{~mol}^{-1}$.

The thermal contributions to the free energy (including the zero-point vibrational energy) were calculated according to the standard ideal-gas/rigid-rotor/harmonic-oscillator approximation at $T=298.15 \mathrm{~K}, p^{\circ}=100 \mathrm{kPa}$. Frequencies were not scaled; however, to avoid spurious large entropy contributions from low-frequency vibrational modes, for which the harmonic-oscillator approximation is unreliable, frequencies below $100 \mathrm{~cm}^{-1}$ were raised (Ribeiro et al., 2011) to $100 \mathrm{~cm}^{-1}$.

$\mathrm{p} K_{\mathrm{a}}$ values were calculated from free energies obtained at the M06-2X/def2-TZVP+ level (Zhao and Truhlar, 2008) using the SMD continuum model for water in Gaussian 09. Frequencies were scaled (Alecu et al., 2010; Kanchanakungwankul et al., 2018) by a factor of 0.972 and raised (Ribeiro et al., 2011) to $\geq 100 \mathrm{~cm}^{-1}$ using the GoodVibes program (Funes-Ardoiz and Paton, 2018) for the calculation of free energies. The $\mathrm{p} K_{\mathrm{a}}$ value of an acid HA was then computed with respect to a reference acid HRef with experimentally known $\mathrm{p} K_{\mathrm{a}}$ as follows:

$$
\mathrm{p} K_{\mathrm{a}}(\mathrm{HA})=\lg (\mathrm{e}) \Delta G_{\mathrm{a}}(\mathrm{HA}) /(R T)+\mathrm{p} K_{\mathrm{a}}^{\text {expt }}(\text { HRef })-\mathrm{p} K_{\mathrm{a}}^{\text {calc }}(\text { HRef })
$$

where $\lg (\mathrm{e})$ is the decadic logarithm of Euler's number, $\Delta G_{\mathrm{a}}(\mathrm{HA})$ $=G^{*}\left(\mathrm{~A}^{-}\right)-G^{*}(\mathrm{HA})$, and $\mathrm{p} K_{\mathrm{a}}^{\text {calc }}($ HRef $)=\lg (\mathrm{e}) \Delta G_{\mathrm{a}}($ HRef $)$.

\section{Choice of Standard States}

We express all thermodynamic quantities in solution with reference to a fixed-solute equal-concentrations standard state as defined by Ben-Naim (Ben-Naim, 1987, 1992), indicated by *, with a concentration of $c^{*}=1 \mathrm{~mol} \mathrm{~L}^{-1}$. The usual gas-phase standard state with $p^{\circ}=100 \mathrm{kPa}$ is indicated by ${ }^{\circ}$; the standard temperature is $T=298.15 \mathrm{~K}$ in both cases. The free energy of solute $S$ in solution contains a liberational term, which replaces the gas-phase translational free energy. In the * standard, these terms are exactly equal,

$$
G_{\text {lib }}^{*}(S, \sin )=G_{\text {trans }}^{*}(S, g) .
$$

The liberational free energy can therefore be calculated as

$$
G_{\text {lib }}^{*}(S, \operatorname{sln})=G_{\text {trans }}^{\circ}(S, g)+\Delta G^{\circ \rightarrow *},
$$

where $\Delta G^{\circ \rightarrow *}=R T \ln \left(R T c^{*} / p^{\circ}\right)=8.0 \mathrm{~kJ} \mathrm{~mol}^{-1}$ is the conversion between standard states, associated with the isothermal compression of $1 \mathrm{~mol}$ of ideal gas from its concentration at $p^{\circ}$ to $c^{*}$. For a process in solution where the number of particles changes, e.g., ion-pairing, the free-energy change $\Delta G_{\mathrm{sln}}{ }^{*}$ therefore includes a term $\Delta G^{\circ \rightarrow *} \times \Sigma_{i} v_{\mathrm{i}}$, where $v_{i}$ are the (signed) stoichiometric coefficients of reactants and products.

\section{RESULTS AND DISCUSSION}

\section{Enhanced Mitochondria-Targeting With a Triphenylphosphonium Carboxylic Acid}

To develop an enhanced mitochondria-targeting head group, we first made a series of alkyltriphenylphosphonium (TPP) salts with a carboxylic acid group on one phenyl group para to the phosphorus (Figure 2A). The uptake of these compounds by energized mitochondria, which will have a large membrane potential, was studied alongside TPP-containing internal standards (IS). Uptake of the IS confirmed that the test compound did not significantly disrupt mitochondrial membrane potential and showed that the mitochondria continued to respond as expected to different additives known to affect the membrane potential. Energized mitochondria were incubated with carboxylic acid $\mathbf{3}$ and IS and uptake of the compounds into mitochondria measured by RP-HPLC (Figure 2B) and quantified (Figure 2C). The IS was taken up by energized mitochondria and abolishing the membrane potential with the uncoupler FCCP (Nicholls and Ferguson, 2013), blocked this uptake (Figures 2B,C). However, carboxylic acid 3 was not taken up by energized mitochondria (Figures 2B,C). A hydrophobicity threshold is required for mitochondrial uptake of alkylTPP molecules (Ross et al., 2006; Finichiu et al., 2015; Hu et al., 2017), but when hydrophobicity was increased by replacing the methyl (3) with a hexyl group (4) there was still no mitochondrial uptake compared to IS (Figure 2D). Incorporating the more hydrophobic dodecyl group (5) led to extensive adsorption to mitochondria that was unaffected by uncoupling with FCCP (Figure 2E). Extending incubation time to $15 \mathrm{~min}$ did not lead to uptake (data not shown). We conclude that alkylTPP salts with a carboxylic acid on one of the phenyl groups of TPP are not taken up by energized mitochondria.

The $\mathrm{pK}_{\mathrm{a}}$ values of 3-5 may have influenced mitochondrial uptake. The enhanced mitochondrial uptake reported previously was for a carboxylic acid attached to a TPP via an undecyl chain (1) with a $\mathrm{pK}_{\mathrm{a}} \sim 4.9$ (Finichiu et al., 2013). Stabilization of the deprotonated form by the phenyl and the phosphonium cation in 3-5 may lower the $\mathrm{pK}_{\mathrm{a}}$, decreasing the proportion in the membrane permeant, protonated form (Figure 1). The $\mathrm{pK}_{\mathrm{a}}$ of 3 was determined computationally by reference to experimental $\mathrm{pK}_{\mathrm{a}}$ values for benzoic acid and phenylacetic acid, giving a $\mathrm{pK}_{\mathrm{a}}$ of $2.5 \pm 0.1$ (Table 1). At intracellular $\mathrm{pH}(7.2)$ the TPP undecylcarboxylic acid $\left(\mathrm{pK}_{\mathrm{a}} \sim 4.9\right)$ (Kanicky and Shah, 2003) would be $\sim 0.5 \%$ in the protonated form, compared to $0.002 \%$ for $3, \sim 300$-fold lower. We next distanced 
TABLE 1 | Calculated $p K_{a}$ values of carboxylic acids $\mathbf{3}, \mathbf{6}$, and $\mathbf{9}$, with reference to benzoic acid and phenylacetic acid.

\begin{tabular}{|c|c|c|c|}
\hline \multirow[t]{2}{*}{ Compound } & \multicolumn{3}{|c|}{$\mathrm{p} K_{\mathrm{a}}$} \\
\hline & $\mathrm{PhCOOH}$ ref & $\begin{array}{c}\mathrm{PhCH}_{2} \mathrm{COOH} \\
\text { ref }\end{array}$ & $\begin{array}{c}\text { Experiment } \\
\text { (Rumble et al. } \\
\text { 2018) }\end{array}$ \\
\hline $\mathrm{PhCOOH}$ & 4.204 & 4.4 & 4.204 \\
\hline $\mathrm{PhCH}_{2} \mathrm{COOH}$ & 4.1 & 4.31 & 4.31 \\
\hline $\mathrm{TPMP}^{+}{ }_{-} \mathrm{COOH}(\mathbf{3})$ & 2.4 & 2.6 & \\
\hline $\mathrm{TPMP}^{+}-\mathrm{CH}_{2} \mathrm{COOH}(\mathbf{6})$ & 2.6 & 2.8 & \\
\hline $\mathrm{TPMP}^{+}-\mathrm{CH}_{2} \mathrm{CH}_{2} \mathrm{COOH}(\mathbf{9})$ & 5.1 & 5.3 & \\
\hline
\end{tabular}

Reference values are printed in italics. the acid group from the phenyl and phosphonium, to make phenylacetic and phenylpropionic derivatives with a range of hydrophobicities (6-11) (Figure 3A). The $\mathrm{pK}_{\mathrm{a}}$ values of 6 and 9 were then computationally determined by reference to the experimental values for benzoic and phenylacetic acid, yielding $\mathrm{pK}_{\mathrm{a}}$ values of $2.7 \pm 0.1$ (6) and $5.2 \pm 0.1$ (9) (Table 1). So, if 3 was excluded from mitochondria by its low $\mathrm{pK}_{\mathrm{a}}$ then 6-8 should also be excluded, while 9-11 should be accumulated. The phenylacetic acid derivatives $\mathbf{6}$ and $\mathbf{7}$ showed no uncoupler-sensitive mitochondrial uptake (Figures 3B,C), while the more hydrophobic $\mathbf{8}$ adsorbed to mitochondria independently of membrane potential (Figure 3D). For the propionic acid derivatives (9-11) we made methyl, butyl and hexyl versions as the hydrophobicity of the dodecyl compounds
A<smiles></smiles>

B
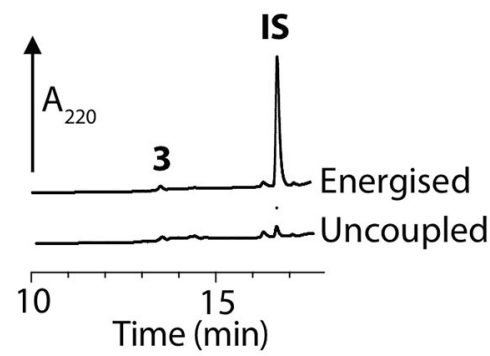

D

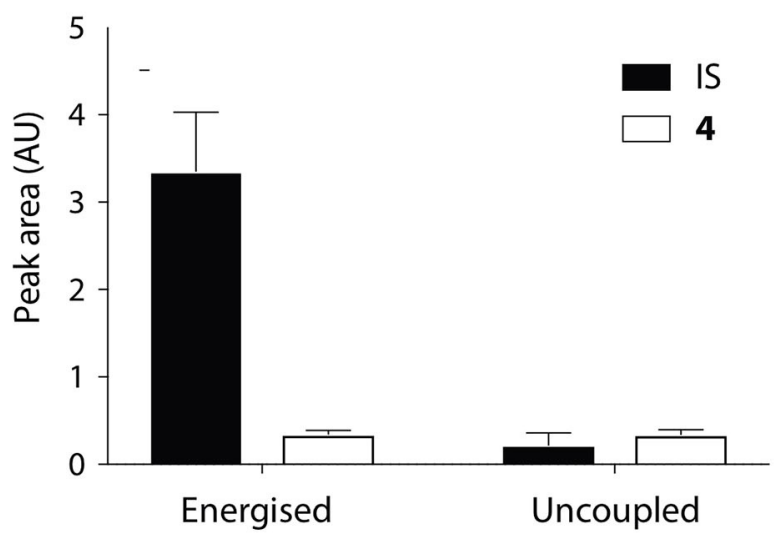<smiles>CCCCCC[Pb](Cl)(Cl)c1ccc(C(=O)O)cc1</smiles>

$\stackrel{\ominus}{\mathrm{Br}}$

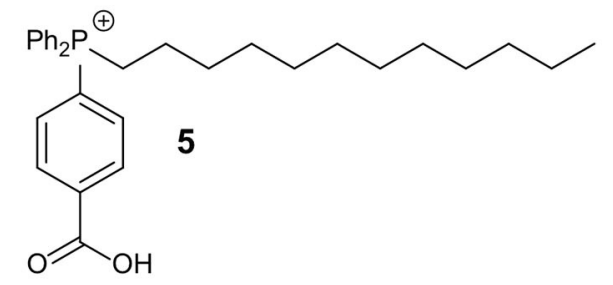

C

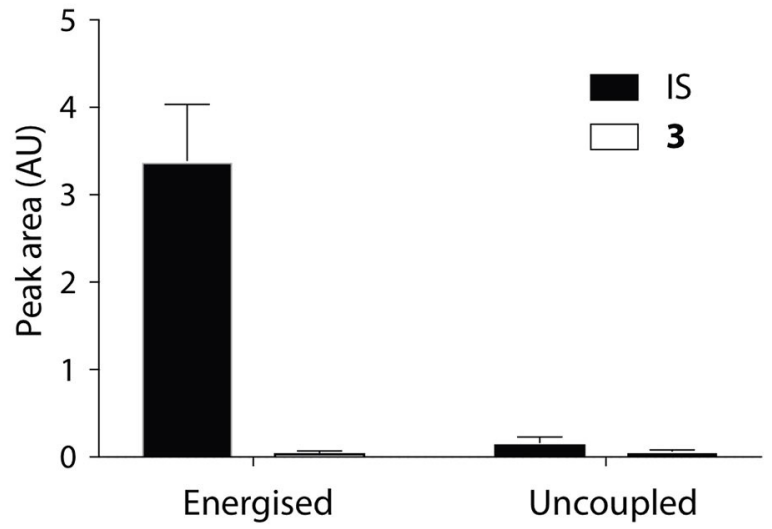

E

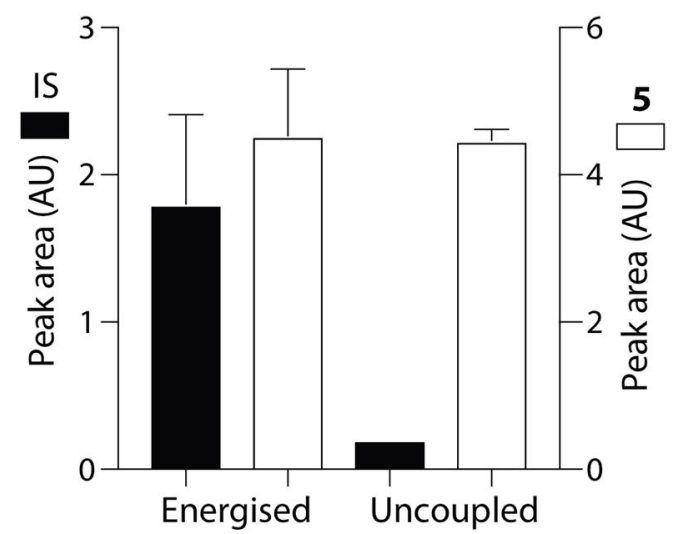

FIGURE 2 | Mitochondrial uptake of TPP benzoic acids. (A) Structure of TPP benzoic acids $\mathbf{3}, \mathbf{4}$, and $\mathbf{5}$. (B) Liver mitochondria (0.5 mg protein/ml) supplemented with succinate and rotenone, were incubated with $\mathbf{3}$ and an internal standard (IS; isoamyl TPP) at $37^{\circ} \mathrm{C}$ for 5 min \pm FCCP. Mitochondria were pelleted by centrifugation and extracted for analysis by RP-HPLC. (C-E) Quantification of mitochondrial uptake of 3, 4, and 5. (C), 3, IS = isoamyl TPP; (D), 4, IS = TPMP; (E), 5, IS = propyITPP). Data are mean \pm S.E.M. of peak areas from three biological replicates. 
A

6<smiles></smiles>

9

$\stackrel{\ominus}{\mathrm{Cl}}$<smiles></smiles>

7

$$
\stackrel{\ominus}{\mathrm{Cl}}
$$<smiles></smiles>

Ci

10<smiles>CCCCCC[Pb]([18OH])c1ccc(CCC(=O)O)cc1</smiles>

8<smiles>[B][Pb]([18OH])(CCCCCCCCCCCC)c1ccc(CC(=O)O)cc1</smiles>

11

Cl<smiles>CCCCCCCCCCCC[Pb]([OH2+])c1ccc(CCC(=O)O)cc1</smiles>

B

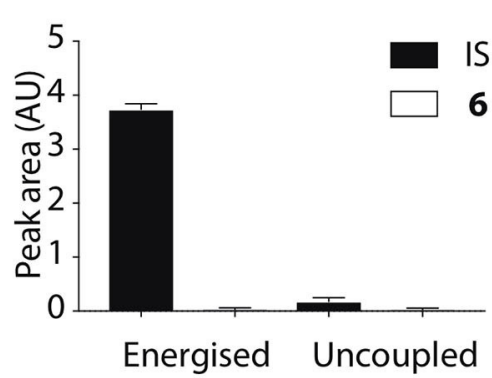

E
C

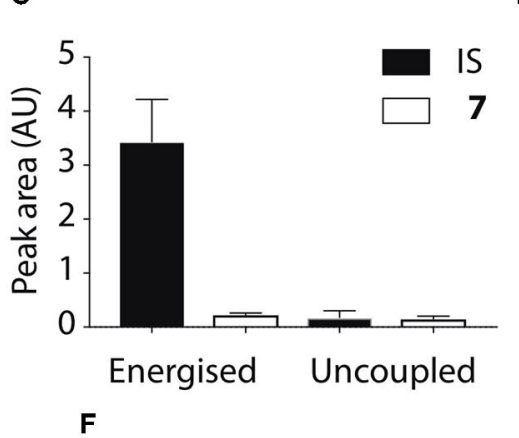

D

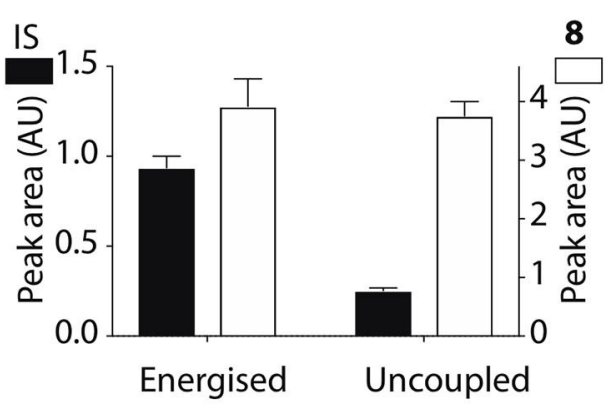

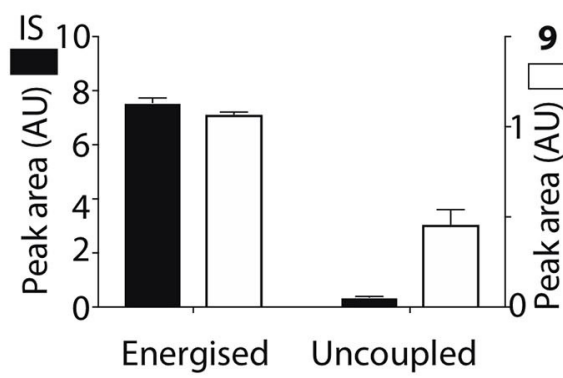

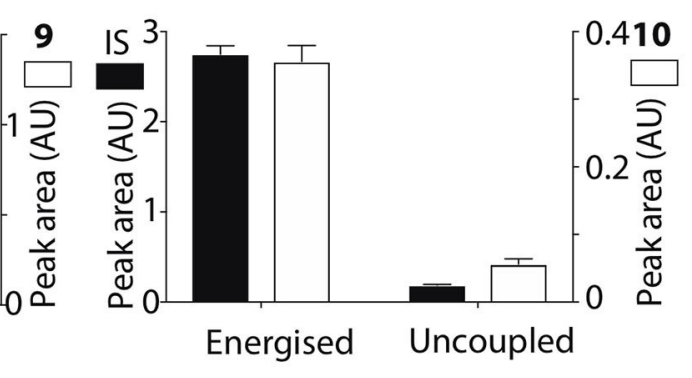

G

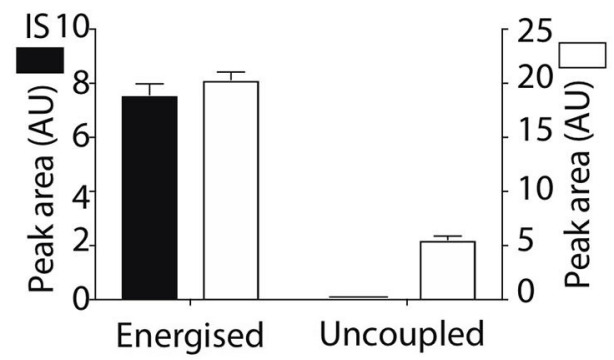

FIGURE 3 | Mitochondrial uptake of TPP acetic and propionic acids. (A) Structure of TPP carboxylic acids 6-11. (B-G) Quantification of mitochondrial uptake of 6-11. Mitochondria were incubated as described in Figure 2B, except for the indicated alterations. (B), 6, IS = isoamylTPP; (C), 7, IS = TPMP; (D), 8, IS = TPMP; (E), $\mathbf{9}(10 \mu \mathrm{M}), \mathrm{IS}=$ propyITPP $(2.5 \mu \mathrm{M})$, and data shown are for incubation with heart mitochondria. The same result was obtained with liver mitochondria; (F), 10, $(10 \mu \mathrm{M})$, IS $=$ TPMP $(5 \mu \mathrm{M})$, and data shown are for liver mitochondria. The same result was obtained with heart mitochondria; (G), 11 , (20 $\mu \mathrm{M})$ IS = TPMP $(2.5 \mu \mathrm{M})$. Data shown are for incubation with heart mitochondria. The same result was obtained with liver mitochondria. Data are mean \pm S.E.M. of RP-HPLC peak areas from three biological replicates except for panel $\mathrm{G}$, where the $+\mathrm{FCCP}$ data are mean \pm range $(n=2)$. 
5 and 8 made mitochondrial experiments uninformative. We found that 9-11 were all taken up by energized mitochondria, but not by uncoupled mitochondria (Figures 3E-G). Thus, the lack of mitochondrial uptake of the benzoic and phenylacetic derivatives was due to their low $\mathrm{pK}_{\mathrm{a}}$. Note that the accumulation of the IS were significantly higher than the carboxylic acids 9-11 (hence two Y-axes are used in Figures), even when the least lipophilic TPP-derivative, TPMP, was used as IS (Figure 3F).

We next assessed whether the mitochondrial uptake of the propionic acid TPP derivatives was enhanced by the $\mathrm{pH}$ gradient as shown in Figure 1. To do this we incubated 10 and TPMP with energized mitochondria and measured the accumulation ratio (ACR), that is the amount of the compound accumulated within mitochondria, normalized to the amount in the medium outside the mitochondria (Finichiu et al., 2013). The ACRs for 10 and TPMP were both substantial and were decreased by uncoupling

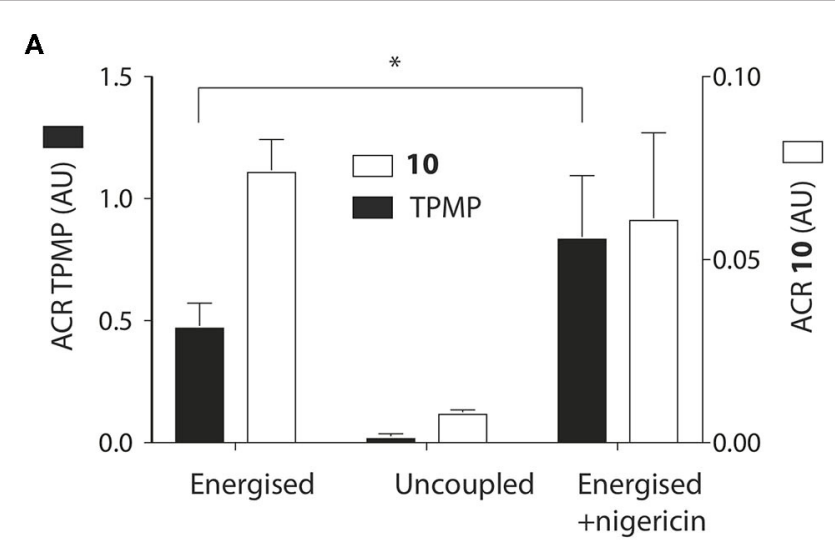

B

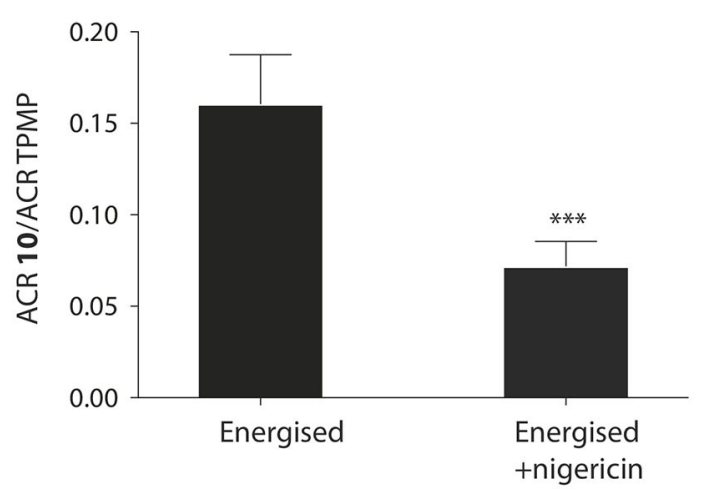

FIGURE 4 | Effect of the pH gradient on mitochondrial uptake of TPP propionic acids. (A) Liver mitochondria (1 mg protein in $1 \mathrm{ml}$ ) were incubated as described in Figure 2B, with $5 \mu \mathrm{M} \mathbf{1 0}$ and TPMP, with FCCP or nigericin as indicated. Then the mitochondria were pelleted and the amounts of $\mathbf{1 0}$ and TPMP in the supernatant and pellets determined by RP-HPLC and used to calculate the ACRs for $\mathbf{1 0}$ and TPMP. (B) The ratios of the ACRs for $\mathbf{1 0}$ and TPMP were determined in the absence and presence of nigericin. Data are mean \pm S.E.M. based on RP-HPLC peak areas from 4 to 5 replicates. ${ }^{*} p<$ $0.05,{ }^{* *} p<0.001$ by Student's unpaired $t$-test. with FCCP (Figure 4A). When the $\mathrm{K}^{+} / \mathrm{H}^{+}$exchanger nigericin (Nicholls and Ferguson, 2013) was added to abolish the $\mathrm{pH}$ gradient and thereby increase the membrane potential, the ACR for TPMP increased as expected, but that for $\mathbf{1 0}$ stayed about the same (Figure 4A). This behavior is consistent with the uptake of 10 being in response to both the membrane potential and the $\mathrm{pH}$ gradient, while that of TPMP is solely determined by the membrane potential (Finichiu et al., 2013). Hence, the addition of nigericin will increase the uptake of TPMP due to the elevation of the membrane potential, but for $\mathbf{1 0}$ the abolition of the $\mathrm{pH}$ gradient will counteract any increased uptake due to an elevated membrane potential. To confirm this, we next compared the ratio of the ACRs for $\mathbf{1 0}$ and TPMP in the presence and absence of nigericin, which showed that nigericin lowered the ratio of these ACRs (Figure 4B). We conclude that the uptake of $\mathbf{1 0}$ into mitochondria is driven by both the membrane potential and the $\mathrm{pH}$ gradient as anticipated in Figure 1.

\section{Utilizing TPP Carboxylic Ester Head Groups to Retain Compounds Within Mitochondria}

The TPP compounds containing benzoic and phenylacetic acid derivatives were not taken up by energized mitochondria because their low $\mathrm{pK}_{\mathrm{a}}$ prevented passage across the mitochondrial inner membrane. However, their esters should be taken up with subsequent enzymatic hydrolysis within mitochondria then generating the membrane-impermeant acids (Figure 5). This would enable the prolonged delivery of drugs and probes to the mitochondrial matrix where enzymatic hydrolysis generates a TPP carboxylic acid "locked" within the mitochondrial matrix (Figure 5). To test this possibility we made ethyl esters (12-15) of the membrane impermeant TPP benzoic and phenylacetic acids while we also modulated the lipophilicity with methyl and hexyl alkyl groups (Figure 6A).

The esters 12-15 were all taken up by energized liver mitochondria and partially hydrolysed within mitochondria (Figures 6B-E), with the benzoate esters 12 and 13 hydrolysing faster than the phenylacetate esters 14 and 15. To further accelerate ester hydrolysis we next made esters (16-19) incorporating the electron-withdrawing trifluoroethyl group (Figure 6F). Trifluoroethyl esters of phenylacetic acid are known to be hydrolysed 40 times more quickly than ethyl esters by the archetypical esterase, pig liver esterase (Barton et al., 1994). All these esters 16-19 were taken up into energized mitochondria and almost completely hydrolysed (Figures 6G-J). The methyl derivatives 12, 16, and 18 were also taken up by energized heart mitochondria where the lower esterase activity enabled both acid and ester to be detected (Figures 6K-M).

Thus, the ethyl and trifluoroethyl esters 12-19 are accumulated by energized mitochondria and are there hydrolysed within the matrix to the corresponding acid. To see if the acid was retained in the matrix, we incubated mitochondria with the esters for $5 \mathrm{~min}$ to generate the acid within the matrix, and then abolished the membrane potential with FCCP. As expected, the IS and the remaining ester 


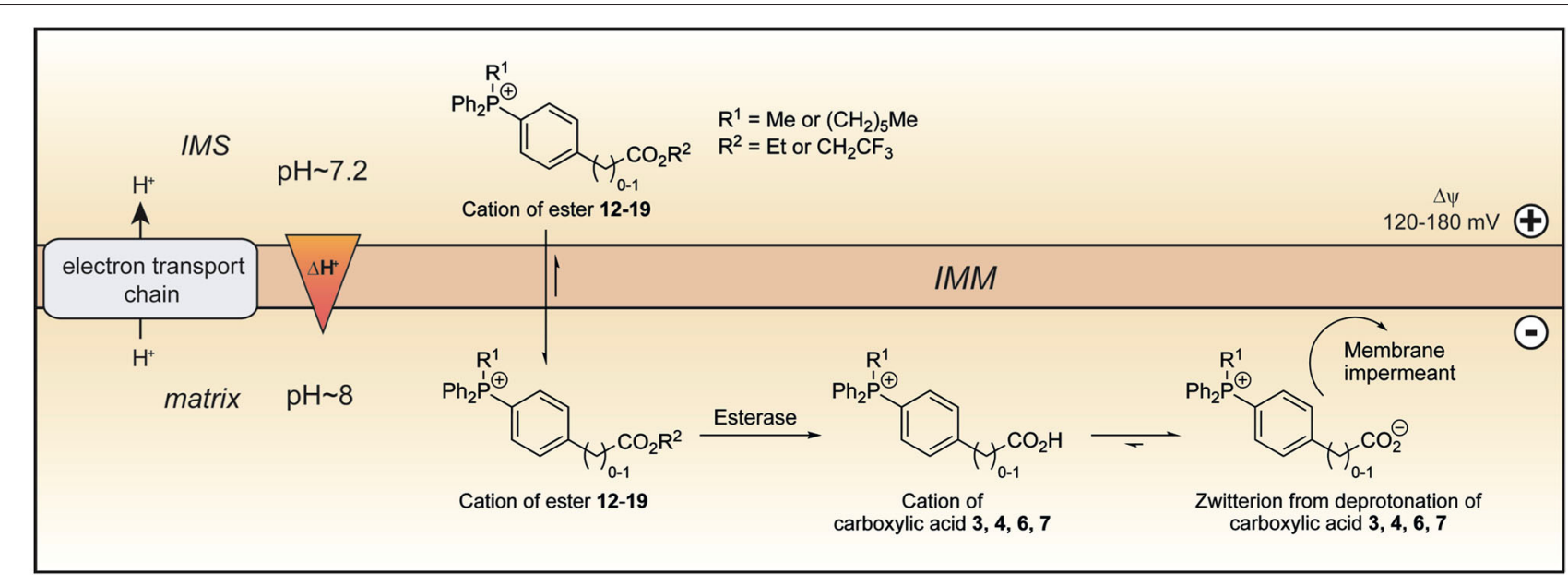

FIGURE 5 | Model of ester uptake and hydrolysis within mitochondria. The lipophilic cations of the esters $\mathbf{1 2 - 1 9}$ readily cross the mitochondrial inner membrane and are accumulated in the matrix in response to the membrane potential. Within mitochondria, hydrolysis forms the cations of the corresponding carboxylic acids, which exist almost exclusively as their highly charged zwitterions at pH 8.0. The zwitterions should be unable to cross the mitochondrial inner membrane and so be trapped within the mitochondrial matrix. IMM, inner mitochondrial membrane; IMS, intermembrane space.

were lost from the uncoupled mitochondria (Figures 7A-D). However, for $\mathbf{1 2}$ and $\mathbf{1 8}$ the acid accumulated within liver or heart mitochondria was also lost upon uncoupling to similar extents as the IS and ester (Figures 7A-D). Esters 13-17 and 19 showed similar behavior (data not shown). FCCP is a lipophilic anion, so to eliminate the possibility that this affected membrane permeability we also used a mixture of mitochondrial inhibitors to abolish the mitochondrial membrane potential. For 12 and 18 (Figures 7E,F) and 14, 16 (data not shown) there was similar loss of the acid upon abolition of the membrane potential as was found with FCCP. Finally, to assess how quickly the acid left mitochondria upon uncoupling we incubated mitochondria with $\mathbf{1 4}$ for $5 \mathrm{~min}$, then added FCCP and then isolated mitochondria at various time points subsequently (Figure 7G). The rate of efflux of the acid was indistinguishable from that of the IS and the ester at the earliest time point accessible in this experiment (Figure 7G). We conclude that these acids rapidly efflux from mitochondria upon loss of the membrane potential.

To see if the acids were retained within mitochondria by a membrane potential we next assessed their retention over time. When mitochondria were incubated with 13, 17 and 19, for up to $15 \mathrm{~min}$ IS accumulation was constant, but the amount of the acid present within mitochondria decreased over time (Figures 7H,I,J), with similar data for esters 12, 13, 16, and 18 (data not shown). To further confirm that acids are lost from energized mitochondria, we incubated mitochondria with 18 for $5 \mathrm{~min}$, and then pelleted the mitochondria and resuspended in fresh incubation medium. The IS was retained by the resuspended mitochondria while the acid was lost (Figure $7 \mathrm{~K}$ ). We conclude that the acids are rapidly lost from energized mitochondria.

The acids 3-8 are not taken up by energized mitochondria, but when generated within mitochondria they are not retained, whether or not there is a membrane potential. One possibility is that the acid can cross the membrane as both the protonated cation and also as the neutral zwitterion. Thus, membrane potential driven uptake of the acids 3-8 in their cationic protonated form, followed by deprotonation and loss of the zwitterion might explain the lack of accumulation. However, this would involve a net influx of protons into the mitochondrial matrix and consequent mitochondrial uncoupling. As increased mitochondrial oxygen consumption is diagnostic of such uncoupling, we measured the effect on this of $\mathbf{3}, \mathbf{4}, \mathbf{6}$, and 7, compared to corresponding methyl or hexyl TPPs controls (Figures 8A,B). In all cases the effect of the acid on oxygen consumption was lower than the control compound, which showed the mild uncoupling expected for TPP-compounds (Reily et al., 2013). We conclude that our data cannot be explained by the acids entering as a cation and leaching out as the neutral zwitterion, unless the membrane potential-driven uptake of the cation is very slow and matched by the efflux of the zwitterion (Figure 8A).

\section{Computational Analysis of Membrane Transport by TPP Derivatives}

Low-p $K_{a}$ TPP-carboxylic acids are not accumulated by energized mitochondria. It is probable that the protonated cation can cross membranes in response to the membrane potential, but that the low concentration of this species renders the flux negligible compared to efflux pathway(s). Once generated within the mitochondrial matrix, the acids are rapidly lost. As this efflux is unaffected by the membrane potential, the acid must cross the membrane as a neutral species. To explore how this might occur we next carried out computational studies to evaluate the Gibbs free energy of membrane crossing of simple salts of TPMP and some of the acids. 
A

12<smiles>CCOC(=O)c1ccc(P(C)(=O)[O-])cc1</smiles>

13<smiles>CCCCCC[Pb]([O-])([O-])c1ccc(C(=O)OCC)cc1</smiles><smiles>CCOC(=O)Cc1ccc(P(C)(=O)O)cc1</smiles><smiles>CCCCCC[Pb]([O-])([O-])c1ccc(CC(=O)OCC)cc1</smiles>

B
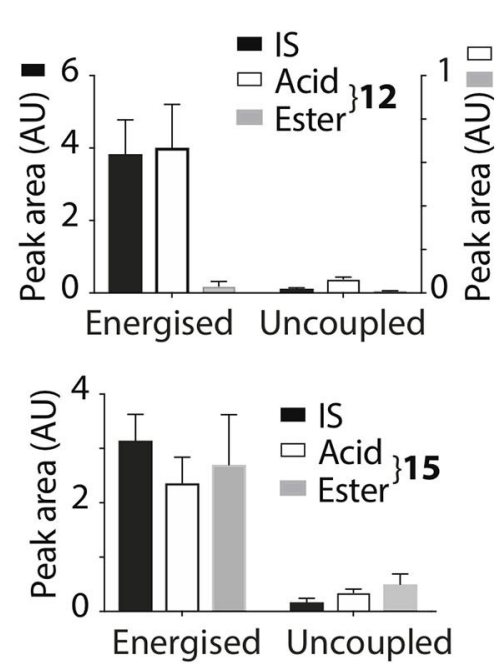

C

D
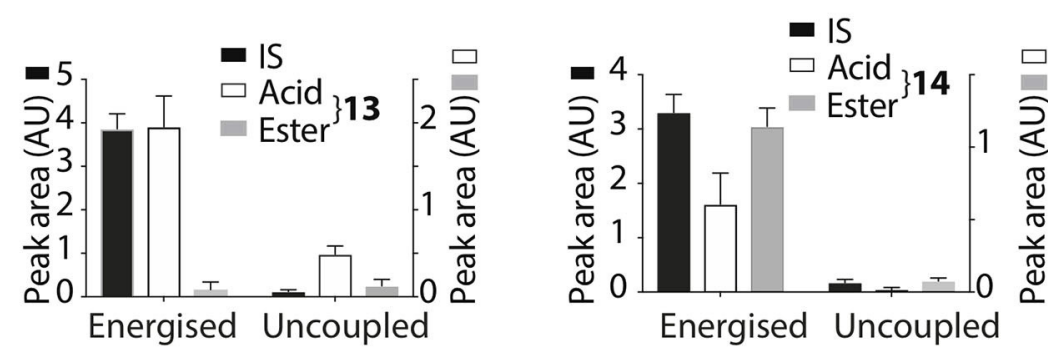

$\mathbf{F}$<smiles>C[Pb]([O-])([O-])c1ccc(C(=O)OCC(F)(F)F)cc1</smiles>

17<smiles>CCCCCC[Pb]([O-])([18OH])c1ccc(C(=O)OCC(F)(F)F)cc1</smiles>

Energised Uncoupled

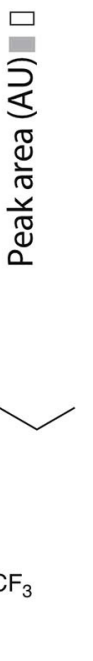

G

H

I

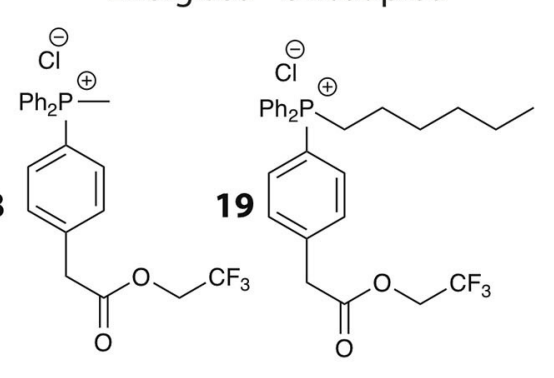

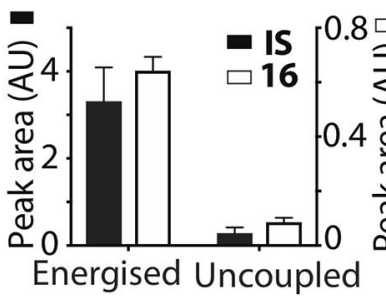

K

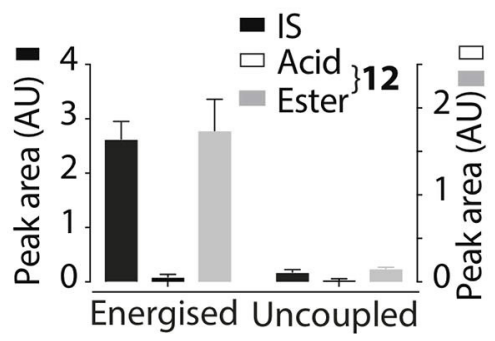

H.

J

Energised Uncoupled

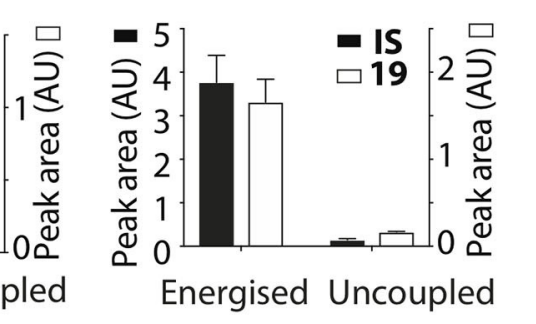

Energised Uncoupled Energised Uncoupled

M

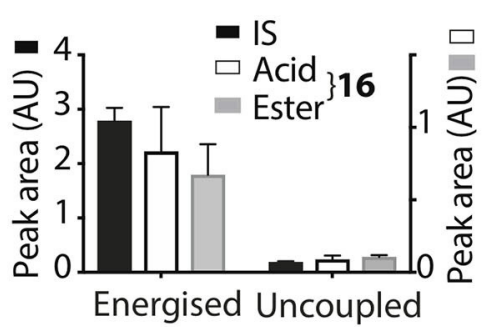

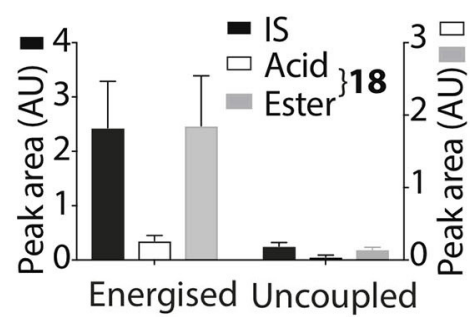

FIGURE 6 | Mitochondrial uptake and hydrolysis of esters. (A) Structures of ethyl esters 12-15 of TPP carboxylic acids. (B-E) Quantification of mitochondrial uptake of 12-15. Liver mitochondria were incubated as described in Figure 2B. (B), 12, IS =isoamylTPP; (C), 13, IS = TPMP; (D), 14, IS = isoamylTPP; (E), 15, IS =TPMP. (F) Structures of trifluoroethyl esters 16-19 of TPP carboxylic acids. (G-J) Liver mitochondria were incubated and analyzed with esters as described in Figure 2B. (G), 16, IS =isoamyITPP; (H), 17, IS = TPMP; (I), 18, IS = TPMP; (J), 19, IS =TPMP. (K-M) Heart mitochondria incubated as described in Figure 2B with 12, 16 or 18 \pm FCCP. (K), 12, IS = isoamylTPP; (L), 16, IS = isoamylTPP; (M), 18, IS = isoamyITPP. Data are mean \pm S.E.M. of RP-HPLC peak areas from three biological replicates. 


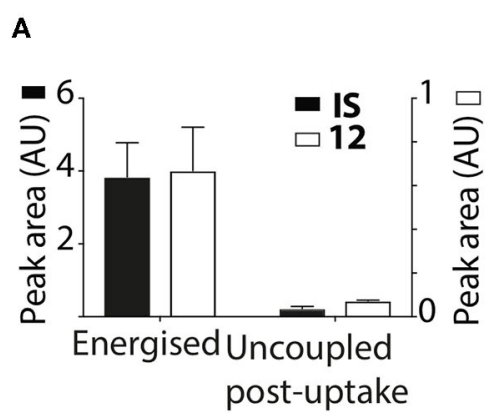

D

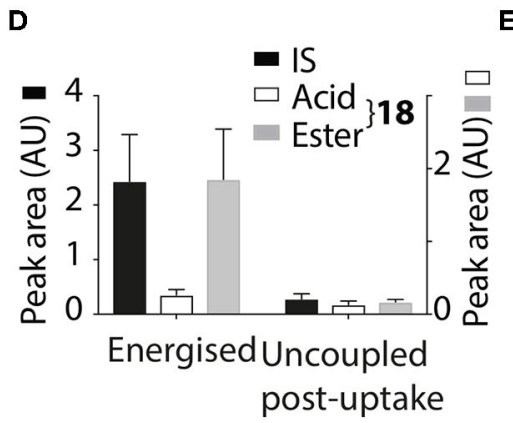

G

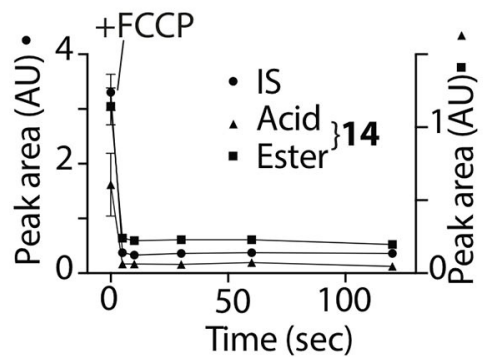

J

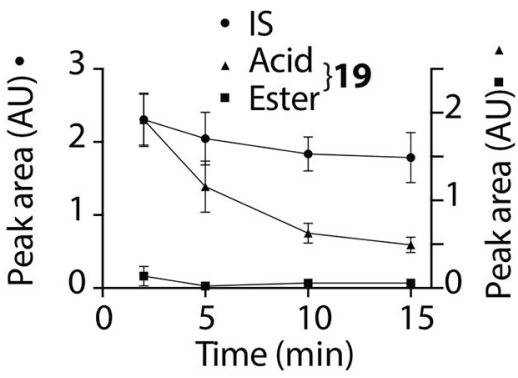

B

E
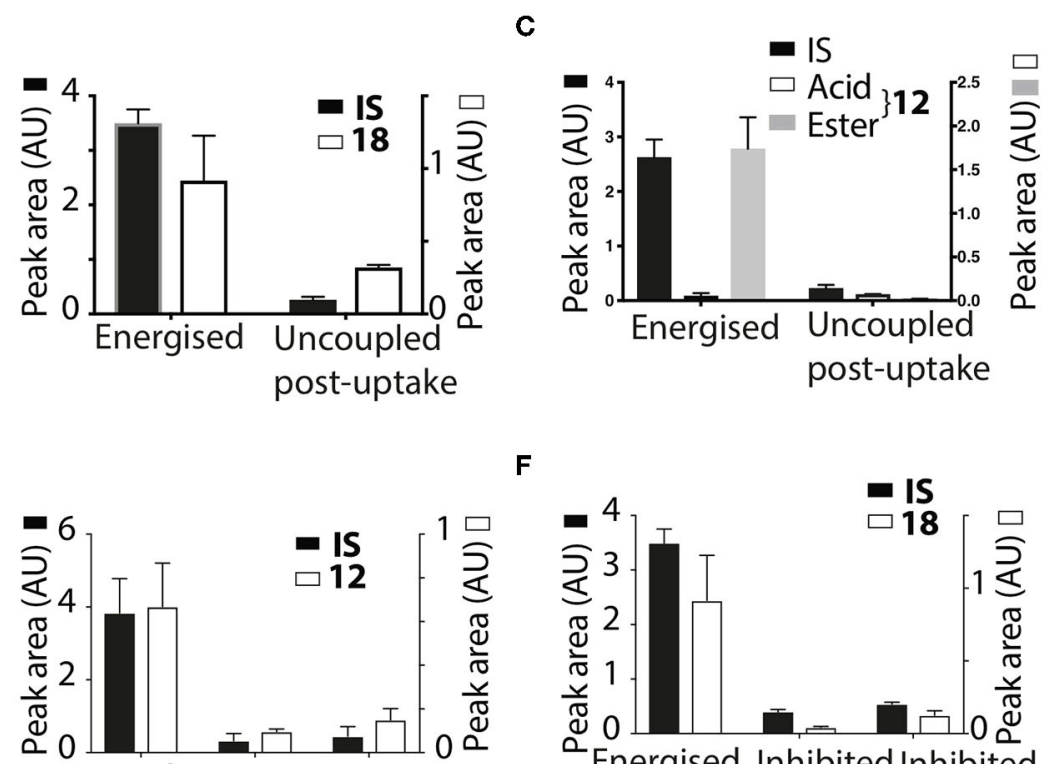

Energised Inhibited Inhibited post-uptake

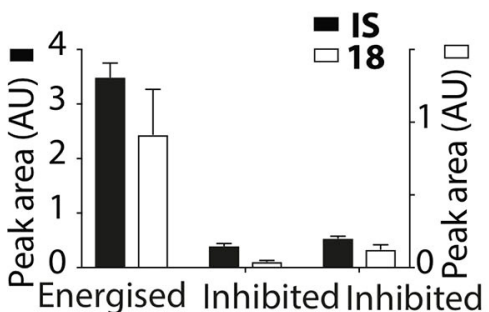

post-uptake

- IS

H 


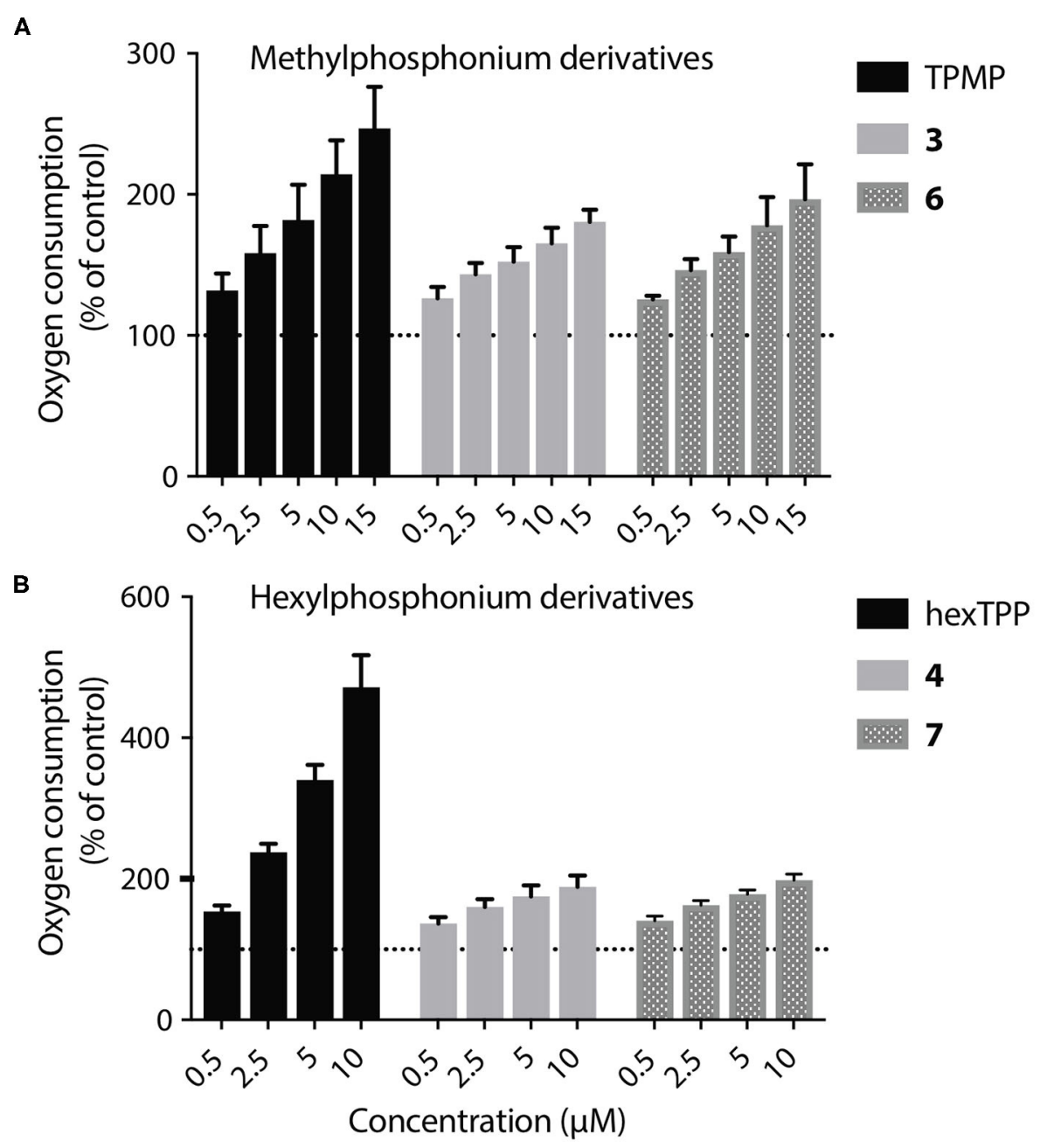

FIGURE 8 | Lack of mitochondrial uncoupling by TPP acids. (A,B) Liver mitochondria $(0.25 \mathrm{mg}$ mitochondrial protein/mL) respiring on succinate were incubated in $\mathrm{KCl}$ buffer $\left(\mathrm{pH} 7.2,37^{\circ} \mathrm{C}\right)$ were in an Oroboros O2K high resolution respirometer A range of concentrations of $\mathbf{3}, \mathbf{4}$, 6, or $\mathbf{7}$, or of appropriate ISs, were incubated and the effect on respiration rate, relative to controls without additions were determined. Data are percentage of initial respiration rate before addition of test compounds and are mean \pm S.E.M. of three biological replicates.

differences in the behavior of cations crossing membranes and accumulating in response to a membrane potential (Robb et al., 2015). It has since received some experimental validation, when (Rokitskaya et al., 2019) showed that calculations on partitioning into an alkane solvent correlate exceptionally well with experiments on transmembrane movement of lipophilic ions across black lipid membranes. The ion can transfer from water to hexane as a free ion, or together with a counter-ion such as $\mathrm{Cl}^{-}$as a charge-neutral ion pair. For the transfer of a single monovalent cation from water to hexane, we write

$$
\mathrm{M}^{+}(\mathrm{aq})+\mathrm{X}^{-}(\mathrm{aq}) \rightarrow \mathrm{M}^{+}(\mathrm{Hex})+\mathrm{X}^{-}(\mathrm{aq})
$$

The biologically relevant counter-anions $\mathrm{X}^{-}$are typically small and strongly hydrophilic, so their partitioning into the apolar phase is negligible and is only shown here to facilitate comparison with the transfer of an ion pair, as is discussed below. The cation is sufficiently lipophilic to transfer to the apolar phase. The change in free energy associated with Equation (1) is therefore the singleion free energy of transfer, $\Delta_{\mathrm{t}} G^{*}\left(\mathrm{M}^{+}\right.$, aq $\rightarrow$ Hex $)$. The transfer free energy of a solute $S$ is defined as the difference in solvation energy of $S$ between the two solvents:

$$
\Delta_{\mathrm{t}} G^{*}(S, \text { aq } \rightarrow \text { Hex })=\Delta_{\text {solv }} G^{*}(S, \operatorname{Hex})-\Delta_{\text {solv }} G^{*}(S, \text { aq })
$$

If we start from the same initial state of free solvated ions, the transfer of a neutral ion pair (IP) contains two components: firstly, the formation of the IP in water and secondly, the transfer of the IP to the apolar phase.

$$
\mathrm{M}^{+}(\mathrm{aq})+\mathrm{X}^{-}(\mathrm{aq}) \rightarrow\left[\mathrm{M}^{+} \cdot \mathrm{X}^{-}\right](\mathrm{aq}) \rightarrow\left[\mathrm{M}^{+} \cdot \mathrm{X}^{-}\right](\mathrm{Hex})
$$

Accordingly, the free-energy change associated with Equation (2) is the sum of the free energies of ion-pairing and of transfer of the 
ion pair.

$$
\Delta_{\mathrm{ip}} G^{*}(\mathrm{aq})+\Delta_{\mathrm{t}} G^{*}(\mathrm{IP}, \mathrm{aq} \rightarrow \mathrm{Hex})
$$

Depending on how well solvated the ion is in the respective phase and the stability of the ion pair, it will be more thermodynamically favorable for an ion to cross either as a free ion (Equation 1) or as an ion pair (Equation 2).

Consider $\mathrm{TPMP}^{+}\left(\mathrm{Ph}_{3} \mathrm{PMe}^{+}\right)$and its ion pair with chloride, $\left[\mathrm{TPMP}^{+} \cdot \mathrm{Cl}^{-}\right.$]: Table 2 lists the calculated free energies of transfer for the free ion and the ion pair; for the ion pair, the free energy of pairing is given together with the sum of pairing + transfer of the pair, which is the relevant free energy for crossing as a pair. Unsurprisingly, the free $\mathrm{TPMP}^{+}$cation is better solvated in water than in hexane, making the transfer from water to hexane thermodynamically unfavorable, albeit far less so than for small ions with localized, concentrated charge, such as $\mathrm{Na}^{+}$. Larger ions, such as $\mathrm{TPMP}^{+}$, have smaller surface charge densities and expose apolar surface patches to the solvent. Hence, they are relatively less well stabilized in water and so the difference between stabilization in the aqueous phase and stabilization by favorable apolar interactions in hexane is less, and the energy cost of transfer from water to hexane is lower (Reichardt and Welton, 2011).

Despite any free ion being strongly solvated in water, the formation of ion pairs in water is only relatively weakly endergonic. All the ion pairs considered here are still more stable in water than in hexane, but their transfer free energies are smaller than for ions in many cases. Generally speaking, the more hydrophilic/polar the free ion is, the more it benefits from ion-pairing in terms of reducing the energetic disadvantage associated with transferring to hexane.
For the example of $\mathrm{TPMP}^{+}$, it transfers relatively easily as a free cation; the transfer as an ion-pair $\left[\mathrm{TPMP}^{+} \cdot \mathrm{Cl}^{-}\right]$is disfavoured by another $14 \mathrm{~kJ} \mathrm{~mol}^{-1}$. In summary, "lipophilic" ions (low overall charge, small surface charge, large apolar surface patches) can transfer as free ions from water to hexane at modest thermodynamic cost. They do not benefit from ion-pairing. On the other hand, for hydrophilic ions (high overall charge, concentrated surface charge, little apolar surface area), it is thermodynamically prohibitive to transfer as free ions. However, when paired with a strongly hydrophilic counter ion the cost of transferring is significantly reduced (Robb et al., 2015).

We now consider derivatives of $\mathrm{TPMP}^{+}$where one of the phenyl rings has been substituted in para position with a carboxyl or carboxymethyl group. There are therefore multiple possibilities of forming ion pairs with counter-anions or cations (Table 2). Comparing the transfer free energies of the single species, $\mathrm{TPMP}^{+}, \mathrm{TPMP}^{+}{ }_{-} \mathrm{COOH}$, and $\mathrm{TPMP}^{+}{ }_{-} \mathrm{COO}^{-}$, it can be seen that introducing the neutral, protonated carboxyl group makes the transfer $\sim 20 \mathrm{~kJ} \mathrm{~mol}^{-1}$ more unfavorable (compared to unsubstituted $\mathrm{TPMP}^{+}$) (Table 2). Transferring the zwitterionic carboxylate is significantly disfavored (by $\sim 40 \mathrm{~kJ} \mathrm{~mol}^{-1}$ ) despite the overall neutral charge because the negative charge of the carboxylate is solvent-exposed and relatively localized. Depending on whether the carboxylic acid is deprotonated or neutral, the following ion pairs were considered: $\left[\mathrm{TPMP}^{+}\right.$$\left.\mathrm{COOH} \cdot \mathrm{Cl}^{-}\right],\left[\mathrm{TPMP}^{+}{ }_{-} \mathrm{COO}^{-} \cdot \mathrm{Cl}^{-}\right]^{-},\left[\mathrm{TPMP}^{+}{ }_{-} \mathrm{COO}^{-}\right.$. $\left.\mathrm{Na}^{+}\right]^{+}$. None of these ion pairs transfers more easily than the corresponding single species (Table 2).

However, the zwitterions $\mathrm{TPMP}^{+}{ }_{-} \mathrm{COO}^{-}$and $\mathrm{TPMP}^{+}$. $\mathrm{CH}_{2} \mathrm{COO}^{-}$have the ability to form self-complementary ("headto-tail") dimers (Figure 9). In these structures, the negatively

TABLE 2 | Calculated free energies of transfer of solute $S$ from water to hexane, $\Delta_{\mathrm{t}} G^{*}\left(S\right.$, aq $\rightarrow$ Hex), and free energies of ion-pairing in water, $\Delta_{i p} G^{*}($ aq).

\begin{tabular}{|c|c|c|c|c|}
\hline Solute $S$ & $\Delta_{\mathrm{t}} G^{\star}(S$, aq $\rightarrow$ Hex $)$ & $\Delta_{\mathrm{ip}} G^{\star}(\mathrm{aq})$ & $\Delta_{\mathrm{ip}} G^{\star}(\mathrm{aq})+\Delta_{\mathrm{t}} G^{\star}(S, \mathrm{aq} \rightarrow \mathrm{Hex})$ & Ion-pair preference \\
\hline TPMP $^{+}$ & 44 & & & \\
\hline $\mathrm{TPMP}^{+}-\mathrm{COOH}$ & 64 & & & \\
\hline $\mathrm{TPMP}^{+}-\mathrm{CH}_{2} \mathrm{COOH}$ & 59 & & & \\
\hline $\mathrm{TPMP}^{+}-\mathrm{CH}_{2} \mathrm{COO}^{-}$ & 88 & & & \\
\hline $\mathrm{TPMP}^{+} \cdot \mathrm{Cl}^{-}$ & 39 & 19 & 58 & 14 \\
\hline $\mathrm{TPMP}^{+}-\mathrm{COOH} \cdot \mathrm{Cl}^{-}$ & 55 & 18 & 73 & 9 \\
\hline $\mathrm{TPMP}^{+}-\mathrm{COO}^{-} \cdot \mathrm{Cl}^{-}$ & 160 & 19 & 179 & 93 \\
\hline $\mathrm{TPMP}^{+}-\mathrm{COO}^{-} \cdot \mathrm{Na}^{+}$ & 126 & -3 & 123 & 40 \\
\hline $\mathrm{TPMP}^{+}-\mathrm{COO}_{2}^{-}{ }^{\mathrm{a}}$ & 39 & 16 & 55 & -30 \\
\hline
\end{tabular}

All free energies are in $\mathrm{kJ} \mathrm{mol}^{-1}$.

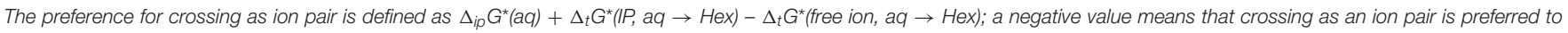
crossing as a free ion. The term "ion-pairing" is used here to designate both the association with a small counter-ion and the dimerisation of zwitterions.

${ }^{a}$ Energies are per mol of monomer.

Comparable values for the transfer of free ions vs. ion-pairs are highlighted in bold. 


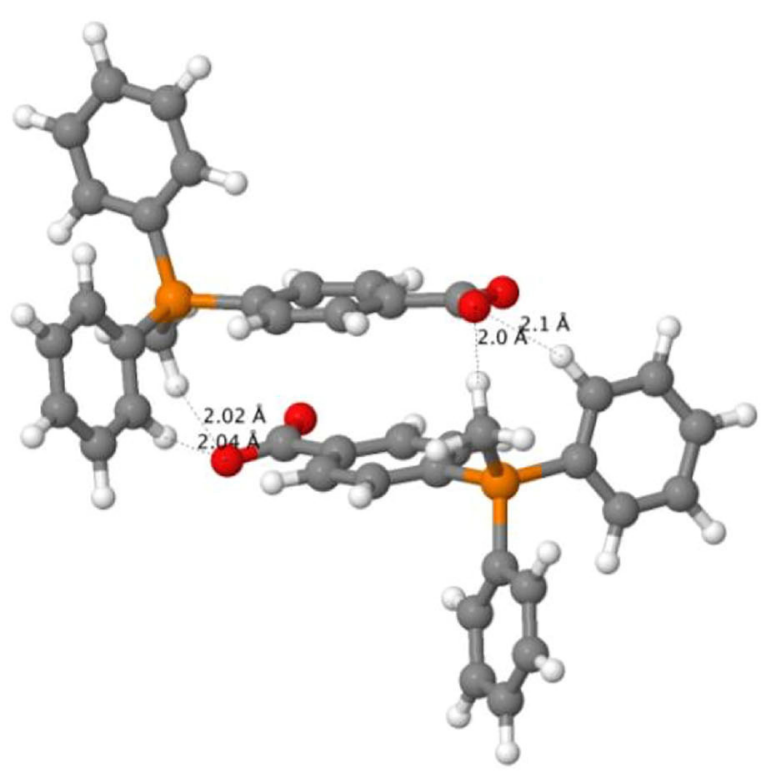

[TPMP+-COO-]2 $\left(\mathrm{H}_{2} \mathrm{O}\right)$

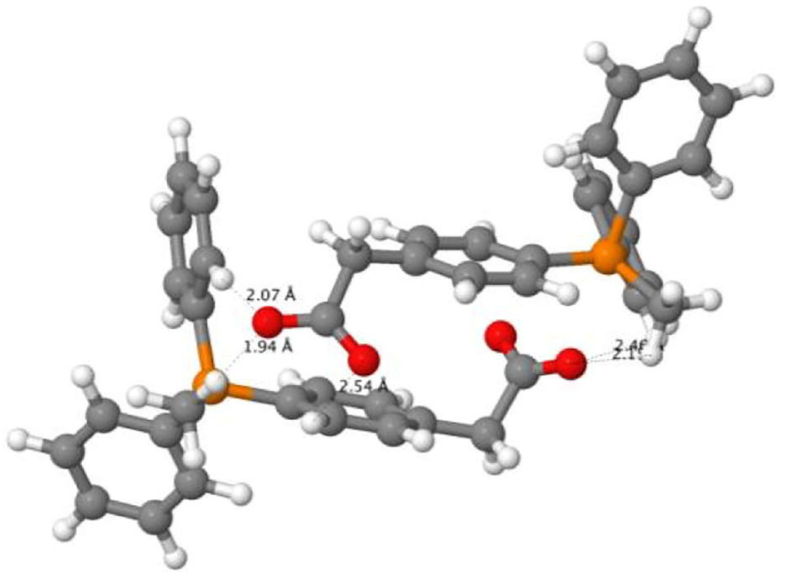

[TPMP+- $\left.\mathrm{CH}_{2} \mathrm{COO}-\right]_{2}\left(\right.$ Conf 1) $\left(\mathrm{H}_{2} \mathrm{O}\right)$

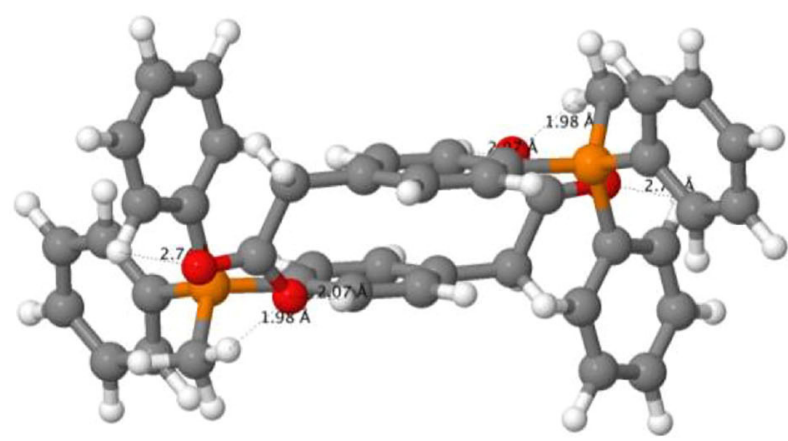

$\left[\mathrm{TPMP}_{+}-\mathrm{CH}_{2} \mathrm{COO}-\right]_{2}(\mathrm{Conf} 2)\left(\mathrm{H}_{2} \mathrm{O}\right)$

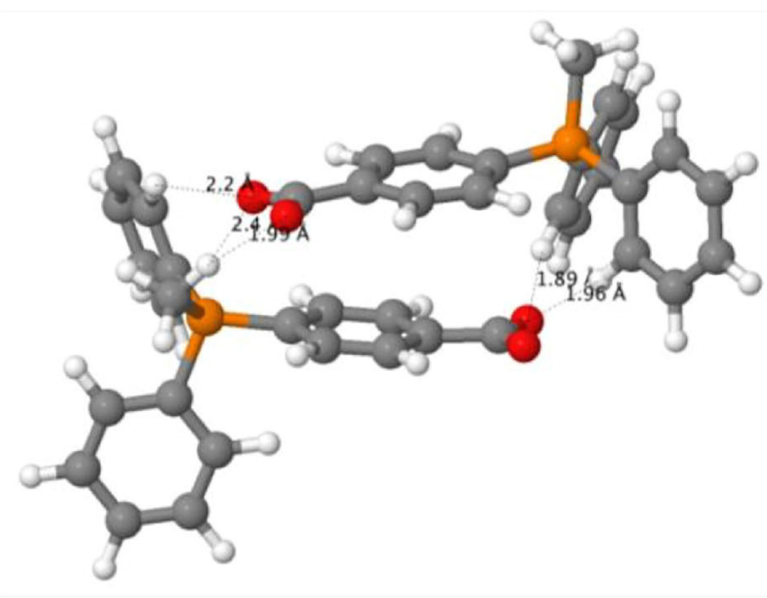

[TPMP+-COO-]2 (Hex)

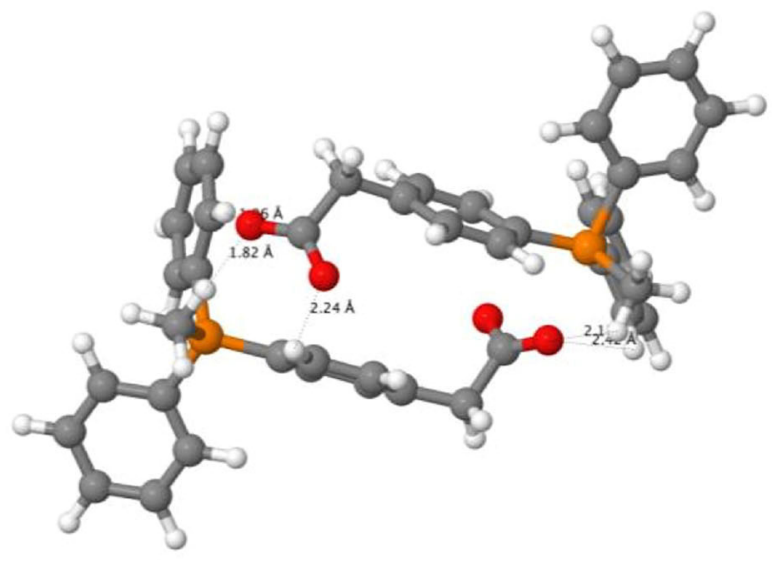

[TPMP+- $\left.\mathrm{CH}_{2} \mathrm{COO}_{-}\right]_{2}($ Conf 1) (Hex)

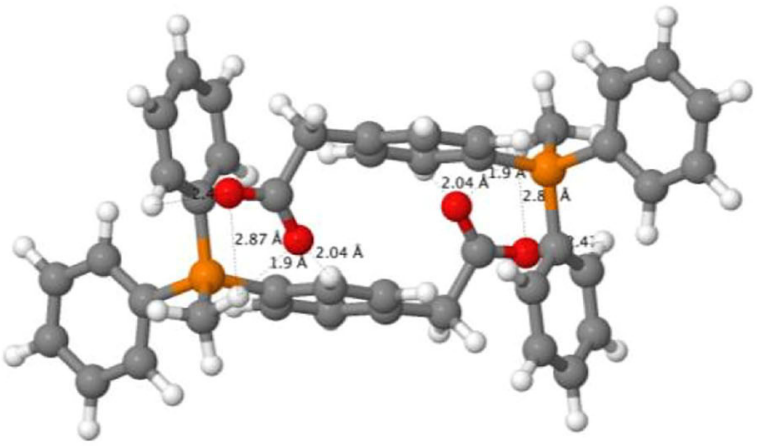

[TPMP $\left.+-\mathrm{CH}_{2} \mathrm{COO}-\right]_{2}($ Conf 2) (Hex) 
A

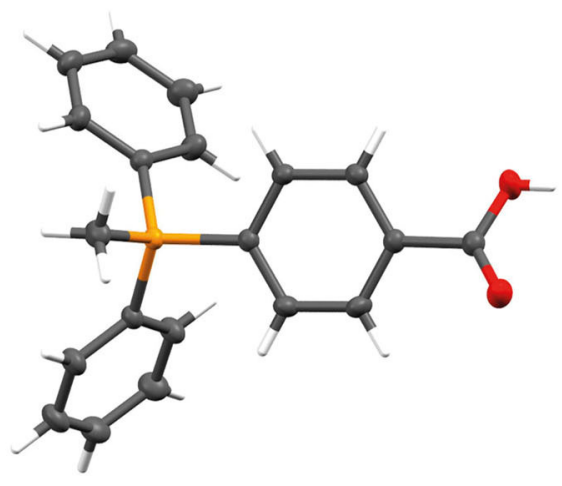

B

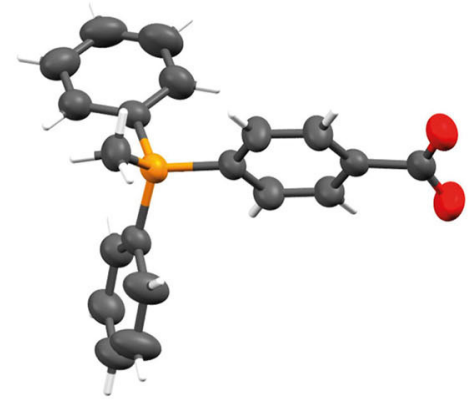

c

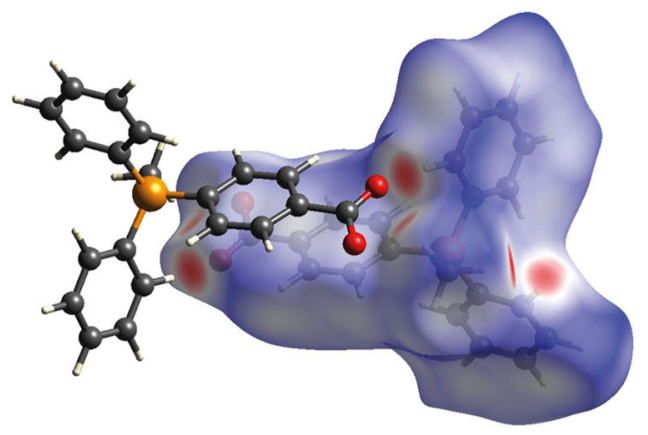

FIGURE 10 | (A) The X-ray crystal structure of $\left[\mathrm{TPMP}^{+}-\mathrm{COOH} \cdot \mathrm{I}^{-}\right]$with atomic displacement ellipsoids drawn at $50 \%$ probability level for non-hydrogen atoms. (B) The X-ray crystal structure of $\mathrm{TPMP}^{+}{ }_{-} \mathrm{COO}^{-}$with atomic displacement ellipsoids drawn at $50 \%$ probability level for non-hydrogen atoms. (C) View of the Hirshfeld surface of $\mathrm{TPMP}^{+} \mathrm{COO}^{-}$mapped with $\mathrm{d}_{\text {norm }}$ property (calculated using CrystalExplorer), contact distance < van der Waals contact are shown in red, equal to it in white and longer as blue. (Spackman and Jayatilaka, 2009).

charged carboxylate groups are partially buried within the pocket formed by the $\alpha$ - and $\beta$-hydrogens of the methyl and phenyl substituents of the phosphonium, respectively, which carry a significant fraction of the positive charge (Figure 9). These stabilizing electrostatic interactions make the dimer formation in water similarly favorable to ion-pairing with a small single ion like $\mathrm{Cl}^{-}$, that is, endergonic by $\sim 15-20 \mathrm{~kJ}$ $\mathrm{mol}^{-1}$. At the same time, in the dimers, the negative charges of the carboxylates are locally compensated for and partially shielded from the solvent so that there is less stabilization from interactions with water. Therefore, transfer to hexane is less unfavorable than for the single zwitterions. For the dimer $\left[\mathrm{TPMP}^{+}-\mathrm{CH}_{2} \mathrm{COO}^{-}\right]_{2}$, we considered two minimum energy structures. Conformer 2 is more stable than Conformer 1 by $45 \mathrm{~kJ} \mathrm{~mol}^{-1}$ in water, but only by $17 \mathrm{~kJ} \mathrm{~mol}^{-1}$ in hexane. Dimerisation in water is therefore more favorable for Conformer 2 , but the transfer energy is less endergonic for Confomer 1 (see Table 2). The two effects largely compensate each other, such that transferring as dimers is clearly preferred for both conformers. Overall, therefore, the dimers transfer significantly more easily than the corresponding single species. The calculated Gibbs energies for all the species taken into consideration (cations, ion pairs, and dimers) are reported in Table 2. We conclude that the most likely way in which the TPP acid derivatives can leave mitochondria is as a neutral dimer of two zwitterionic molecules (Figure 9).

\section{X-ray Crystallography Support for Dimers}

Finally, we sought crystallographic evidence of the benefit of the interactions identified in our models of the dimer $\left[\mathrm{TPMP}^{+}-\mathrm{COO}^{-}\right]_{2}$. To do this, we synthesized the pure zwitterionic species without any counterion. First, the iodide salt was prepared by the method used to make the chloride salt 3 of the carboxylic acid (Scheme 1), but without ion exchange. Single crystals of this compound, $\left[\mathrm{TPMP}^{+} \mathrm{COOH}\right.$ . $\mathrm{I}^{-}$, were grown. Treatment of the iodide salt with silver carbonate removed the iodide and allowed single crystals of the zwitterion, $\mathrm{TPMP}^{+}{ }_{-} \mathrm{COO}^{-}$, also to be grown. Single crystal Xray diffraction revealed the structures of both $\left[\mathrm{TPMP}^{+}-\mathrm{COOH}\right.$ . $\left.\mathrm{I}^{-}\right]$and $\mathrm{TPMP}^{+}{ }_{-} \mathrm{COO}^{-}$(Figures 10A,B). $\left[\mathrm{TPMP}^{+}{ }_{-} \mathrm{COOH}\right.$ - $\mathrm{I}^{-}$] has a $\mathrm{C}-\mathrm{OH}$ length of $1.325(2) \AA$ and a $\mathrm{C}=\mathrm{O}$ length of $1.204(2) \AA$, which are close to the typical C-O (1.308 $\AA$ ) and $\mathrm{C}=\mathrm{O}(1.214 \AA)$ bond lengths in carboxylic acids (Allen et al., 2006). As expected, there is no halogen present in the structure of $\mathrm{TPMP}^{+}-\mathrm{COO}^{-}$with the positive charge of the phosphonium ions balanced in the crystal by the negatively charged carboxylate ions. The $\mathrm{C}-\mathrm{O}$ bond lengths of 1.233(3) and 1.246(3) $\AA$ match closely the typical bond length of $1.254 \AA$ for a delocalised $\mathrm{C}=\mathrm{O}$ carboxylate bond (Allen et al., 2006). Within the crystal lattice multiple individual molecules interact to satisfy the attractive electrostatic interactions in a range of packing motifs. Among these, evidence for the proposed "head-to-tail" dimers was observed (Figure 10C). 


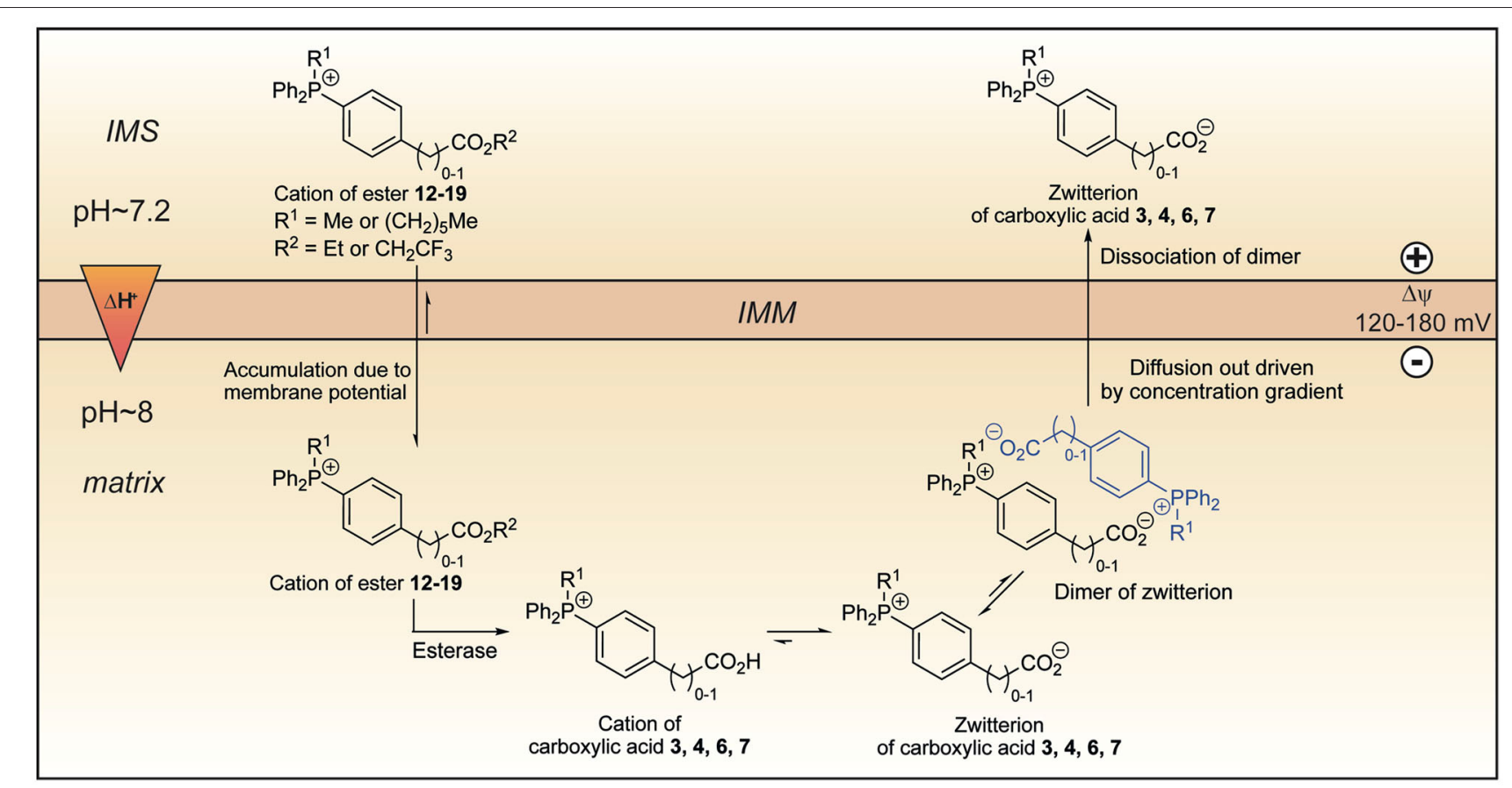

FIGURE 11 | Model explaining the behavior of the esters 12-19 within mitochondria. Lipophilic cations of esters 12-19 cross the mitochondrial inner membrane and accumulate several hundred-fold due to membrane potential. Hydrolysis forms the cations of carboxylic acids $\mathbf{3}, \mathbf{4}, \mathbf{6}$, and $\mathbf{7}$ which exist almost exclusively as their highly charged zwitterions at $\mathrm{pH}$ 8.0. Two molecules of the zwitterions dimerise forming an ion couple that diffuses out driven by the concentration gradient created by accumulation of the esters 12-19 and then dissociate. IMM, inner mitochondrial membrane; IMS, intermembrane space.

Here, the carboxylate group of one molecule is in close proximity to the $\alpha$-hydrogen of the methyl (2.440 $\AA$ ) and the $\beta$ hydrogen of the phenyl (2.237 $\AA$ ) of its neighbor's phosphonium group. These distances agree well with those predicted in our theoretical models of the dimer $\left[\mathrm{TPMP}^{+}-\mathrm{CH}_{2} \mathrm{COO}^{-}\right]_{2}$ (Figure 9).

\section{CONCLUSION}

We set out to develop a mitochondria-targeting strategy that would enhance uptake into mitochondria using a targeting head group that would increase mitochondrial uptake of simple TPP cations by responding not only to the membrane potential but also to the $\mathrm{pH}$ gradient across the mitochondrial inner membrane. Here we show that incorporation of a propionic acid into a TPP moiety provides derivatives that accumulate in response to both these factors, albeit with a lower ACR than simple TPP compounds. On the other hand, we found that TPP moieties derivatised with benzoic or phenylacetic acid groups on the phosphonium were not taken up by mitochondria due to the low $\mathrm{pK}_{\mathrm{a}}$ of these acids depleting the amount of cation available to cross the membrane. This suggested that this finding could be exploited to generate esters that would generate such membrane impermeant acids within the mitochondrial matrix thereby locking molecules within the mitochondrial matrix. As the ability to lock molecules within mitochondria might facilitate the development of more persistent mitochondrial probes and therapies, we explored this possibility. To our surprise, we found that despite their low $\mathrm{pK}_{\mathrm{a}}$ these acids were rapidly lost from mitochondria, irrespective of the presence of a membrane potential. A computational approach suggested that this occurred by the zwitterions forming dimers and that these neutral dimers were then able to pass through the mitochondrial inner membrane. Thus, esters 1219 accumulate in the matrix driven by the membrane potential, but upon hydrolysis, the corresponding carboxylic acids 3, 4, 6, and 7 are not trapped (Figure 11). Instead, they diffuse out of the matrix as dimers of their zwitterions, driven by the concentration gradient. Our work suggests that making TPP carboxylic compounds in which the ability to form dimers was prevented (e.g., by incorporating bulky groups) would improve delivery and open up a new mitochondrial lock in strategy.

In summary, by investigating methods to enhance TPP delivery to mitochondria we have revealed the factors that govern uptake of TPP-carboxylic acids and exposed new potential strategies, which we will explore in future work.

\section{DATA AVAILABILITY STATEMENT}

The NMR data, the Fourier transformed NMR spectra for the synthesised compounds and xyz coordinates and energies of all optimised structures can be found in the University of Glasgow repository http://dx.doi.org/10.5525/gla.researchdata.1004. The 
CIF files CCDC 1998454 [TPMP $^{+}{ }_{-} \mathrm{COO}^{-}$] and 1998455 $\left(\mathrm{TPMP}^{+}{ }_{-} \mathrm{COOH} . \mathrm{I}^{-}\right.$] contain the supplementary crystallographic data for this paper. These data can be obtained free of charge from The Cambridge Crystallographic Data Centre via www.ccdc.cam. ac.uk/structure.

\section{ETHICS STATEMENT}

All experiments were conducted according to the Animals Scientific Procedures Act 1986 (UK) and directive 2010/63/EU of the European Parliament guidelines on the protection of animals used for scientific purposes. All experiments were approved by the University of Cambridge Institutional Animal Welfare and Ethical Review Body.

\section{AUTHOR CONTRIBUTIONS}

LP synthesized the majority of the compounds and carried out most biological testing. HS carried out the computational calculations. SC synthesized some of the compounds. SW and CW carried out and interpreted the single X-ray crystallography study. TP and TB carried out biological testing. HP helped design of biological experiments. $\mathrm{RH}$ and $\mathrm{MM}$ supervised the project

\section{REFERENCES}

Ahlrichs, R., Bär, M., Häser, M., Horn, H., and Kölmel, C. (1989). Electronic structure calculations on workstation computers: the program system turbomole. Chem. Phys. Lett. 162, 165-169. doi: 10.1016/0009-2614(89) 85118-8

Alecu, I. M., Zheng, J., Zhao, Y., and Truhlar, D. G. (2010). Computational thermochemistry: scale factor databases and scale factors for vibrational frequencies obtained from electronic model chemistries. J. Chem. Theory Comput. 6, 2872-2887. doi: 10.1021/ct100326h

Allen, F. H., Watson, D. G., Brammer, L., Orpen, A. G., and Taylor, R. (2006). Typical interatomic distances: organic compounds. Int. Tables Crystallog. C 9, 790-811. doi: 10.1107/9780955360206000062

Barton, P., Laws, A. P., and Page, M. I. (1994). Structure-activity relationships in the esterase-catalysed hydrolysis and transesterification of esters and lactones. J. Chem. Soc, Perkin Trans. 2, 2021-2029. doi: 10.1039/P29940002021

Ben-Naim, A. (1987). Solvation Thermodynamics. New York, NY: Plenum Press.

Ben-Naim, A. (1992). Statistical Thermodynamics for Chemists and Biochemists. New York, NY: Plenum Press.

Chappell, J. B., and Hansford, R. G. (1972). "Preparation of mitochondria from animal tissues and yeasts," in Subcellular Components: Preparation and Fractionation, ed. G. Birnie (London: Butterworths), 77-91.

Dydio, P., Detz, R. J., de Bruin, B., and Reek, J. N. (2014). Beyond classical reactivity patterns: hydroformylation of vinyl and allyl arenes to valuable beta- and gamma-aldehyde intermediates using supramolecular catalysis. J. Am. Chem. Soc. 136, 8418-8429. doi: 10.1021/ja503033q

Finichiu, P. G., James, A. M., Larsen, L., Smith, R. A., and Murphy, M. P. (2013). Mitochondrial accumulation of a lipophilic cation conjugated to an ionisable group depends on membrane potential, $\mathrm{pH}$ gradient and $\mathrm{pK}_{\mathrm{a}}$ : implications for the design of mitochondrial probes and therapies. J. Bioenerg. Biomembr. 45, 165-173. doi: 10.1007/s10863-012-9493-5

Finichiu, P. G., Larsen, D. S., Evans, C., Larsen, L., Bright, T. P., Robb, E. L., et al. (2015). A mitochondria-targeted derivative of ascorbate: MitoC. Free Radic. Biol. Med. 89, 668-678. doi: 10.1016/j.freeradbiomed.2015.07.160

Frisch, M. J., Trucks, G. W., Schlegel, H. B., Scuseria, G. E., Robb, M. A., and Cheeseman, J. R. (2013). Gaussian 09. Version Revision E.01. Wallingford, CT: Gaussian, Inc. and the manuscript writing. All authors contributed to the article and approved the submitted version.

\section{FUNDING}

Work in the MM laboratory was supported by the Medical Research Council UK (MC_U105663142) and by a Wellcome Trust Investigator award (110159/A/15/Z). Work in the RH laboratory was supported by a Wellcome Trust Investigator award (WT110158/Z/15/Z) and LP was supported by a studentship from the University of Glasgow.

\section{ACKNOWLEDGMENTS}

HS is grateful to Kristiāns Čerņevičs for performing some of the calculations reported here as part of his MSci thesis at the University of Glasgow.

\section{SUPPLEMENTARY MATERIAL}

The Supplementary Material for this article can be found online at: https://www.frontiersin.org/articles/10.3389/fchem. 2020.00783/full\#supplementary-material

Funes-Ardoiz, I., and Paton, R. S. (2018). GoodVibes V. 2.0.3. doi: 10.5281/zenodo.1435820

Gorman, G. S., Chinnery, P. F., DiMauro, S., Hirano, M., Koga, Y., McFarland, R., et al. (2016). Mitochondrial diseases. Nat. Rev. Dis. Primers. 2:16080. doi: 10.1038/nrdp.2016.80

Grimme, S., Antony, J., Ehrlich, S., and Krieg, H. (2010). A consistent and accurate $\mathrm{ab}$ initio parametrization of density functional dispersion correction (DFT-D) for the 94 elements H-Pu. J. Chem. Phys. 132:154104. doi: 10.1063/1.3382344

Grimme, S., Ehrlich, S., and Goerigk, L. (2011). Effect of the damping function in dispersion corrected density functional theory. J. Comput. Chem. 32, 1456-1465. doi: 10.1002/jcc.21759

Häser, M., and Ahlrichs, R. (1989). Improvements on the direct SCF method. J. Comput. Chem. 10, 104-111. doi: 10.1002/jcc.540100111

Hu, Z., Sim, Y., Kon, O. L., Ng, W. H., Ribeiro, A. J., Ramos, M. J., et al. (2017). Unique triphenylphosphonium derivatives for enhanced mitochondrial uptake and photodynamic therapy. Bioconjugate Chem. 28, 590-599. doi: 10.1021/acs.bioconjchem.6b00682

Hünenberger, P., and Reif, M. (2011). Single-Ion Solvation: Experimental and Theoretical Approaches to Elusive Thermodynamic Quantities. London: Royal Society of Chemistry.

Jean, S. R., Ahmed, M., Lei, E. K., Wisnovsky, S. P., and Kelley, S. O. (2016). Peptide-mediated delivery of chemical probes and therapeutics to mitochondria. Acc. Chem. Res. 49, 1893-1902. doi: 10.1021/acs.accounts.6b00277

Kanchanakungwankul, S., Bao, J. L., Zheng, J., Alecu, I. M., Lynch, B. J., Zhao, Y., et al. (2018). Database of Frequency Scale Factors for Electronic Model Chemistries. 4th Edn. Available online at: https://comp.chem.umn.edu/ freqscale/

Kanicky, J. R., and Shah, D. O. (2003). Effect of premicellar aggregation on the pKa of fatty acid soap solutions. Langmuir 19, 2034-2038. doi: 10.1021/la020672y

Klamt, A. (1996). Calculation of UV/Vis spectra in solution. J. Phys. Chem. 100, 3349-3353. doi: 10.1021/jp950607f

Klamt, A., and Jonas, V. (1996). Treatment of the outlying charge in continuum solvation models. J. Chem. Phys. 105, 9972-9981. doi: 10.1063/1.472829

Klamt, A., and Schüürmann, G. (1993). COSMO-A new approach to dielectric screening in solvents with explicit expressions for the screening energy and its gradient. J. Chem. Soc. Perkin Trans. 2, 799-805. doi: 10.1039/P29930000799 
Logan, A., Cocheme, H. M., Li Pun, P. B., Apostolova, N., Smith, R. A., Larsen, L., et al. (2014). Using exomarkers to assess mitochondrial reactive species in vivo. Biochim. Biophys. Acta 1840, 923-930. doi: 10.1016/j.bbagen.2013.05.026

Marenich, A. V., Cramer, C. J., and Truhlar, D. G. (2009). Universal solvation model based on solute electron density and on a continuum model of the solvent defined by the bulk dielectric constant and atomic surface tensions. J. Phys. Chem. B 113, 6378-6396. doi: 10.1021/jp810292n

Murphy, M. P., and Hartley, R. C. (2018). Mitochondria as a therapeutic target for common pathologies. Nat. Rev. Drug Discov. 17, 865-886. doi: $10.1038 / \mathrm{nrd} .2018 .174$

Nicholls, D. G., and Ferguson, S. J. (2013). Bioenergetics 4. London: Academic Press.

Nunnari, J., and Suomalainen, A. (2012). Mitochondria: in sickness and in health. Cell 148, 1145-1159. doi: 10.1016/j.cell.2012.02.035

Qin, L., Hu, B., Neumann, K. D., Linstad, E. J., McCauley, K., Veness, J., et al. (2015). A mild and general one-pot synthesis of densely functionalized diaryliodonium salts. Eur. J. Org. Chem. 2015, 5919-5924. doi: 10.1002/ejoc.201500986

Reichardt, C., and Welton, T. (2011). Solvents and Solvent Effects in Organic Chemistry, 4th Edn. Weinheim: Wiley-VCH.

Reily, C., Mitchell, T., Chacko, B. K., Benavides, G. A., Murphy, M. P., and DarleyUsmar, V. M. (2013). Mitochondrially targeted compounds and their impact on cellular bioenergetics. Redox Biol. 1, 86-93. doi: 10.1016/j.redox.2012. 11.009

Ribeiro, R. F., Marenich, A. V., Cramer, C. J., and Truhlar, D. G. (2011). Use of solution-phase vibrational frequencies in continuum models for the free energy of solvation. J. Phys. Chem. B. 115, 14556-14562. doi: 10.1021/jp2 $05508 \mathrm{z}$

Robb, E. L., Gawel, J. M., Aksentijevic, D., Cocheme, H. M., Stewart, T. S., Shchepinova, M. M., et al. (2015). Selective superoxide generation within mitochondria by the targeted redox cycler MitoParaquat. Free Radic. Biol. Med. 89, 883-894. doi: 10.1016/j.freeradbiomed.2015.08.021

Rokitskaya, T. I., Luzhkov, V. B., Korshunova, G. A., Tashlitsky, V. N., and Antonenko, Y. N. (2019). Effect of methyl and halogen substituents on the transmembrane movement of lipophilic ions. Phys. Chem. Chem. Phys. 21, 23355-23363. doi: 10.1039/c9cp03460a

Ross, M. F., Da Ros, T., Blaikie, F. H., Prime, T. A., Porteous, C. M., Severina, I. I., et al. (2006). Accumulation of lipophilic dications by mitochondria and cells. Biochem. J. 400, 199-208. doi: 10.1042/BJ20060919

Rumble, J. R., Lide, D. R., Bruno, T. J. (eds.) (2018). CRC Handbook of Chemistry and Physics. 99th edn. Boca Raton, FL: CRC Press.

Sierka, M., Hogekamp, A., and Ahlrichs, R. (2003). Fast evaluation of the Coulomb potential for electron densities using multipole accelerated resolution of identity approximation. J. Chem. Phys. 118, 9136-9148. doi: 10.1063/1.15 67253

Smith, R. A., Hartley, R. C., Cocheme, H. M., and Murphy, M. P. (2012). Mitochondrial pharmacology. Trends Pharmacol. Sci. 33, 341-352. doi: 10.1016/j.tips.2012.03.010
Smith, R. A., Hartley, R. C., and Murphy, M. P. (2011). Mitochondria-targeted small molecule therapeutics and probes. Antioxid. Redox Sign. 15, 3021-3038. doi: 10.1089/ars.2011.3969

Smith, R. A., Porteous, C. M., Gane, A. M., and Murphy, M. P. (2003). Delivery of bioactive molecules to mitochondria in vivo. Proc. Natl. Acad. Sci. U.S.A. 100, 5407-5412. doi: 10.1073/pnas.0931245100

Spackman, M. A., and Jayatilaka, D. (2009). Hirshfeld surface analysis Cryst. Eng. Comm. 11, 19-32. doi: 10.1039/B818330A

Tao, J. M., Perdew, J. P., Staroverov, V. N., and Scuseria, G. E. (2003). Climbing the density functional ladder: nonempirical meta-generalized gradient approximation designed for molecules and solids. Phys. Rev. Lett. 91, 146401. doi: 10.1103/PhysRevLett.91.146401

Tomasi, J., Mennucci, B., and Cammi, R. (2005). Quantum mechanical continuum solvation models. Chem. Rev. 105, 2999-3093. doi: 10.1021/cr9904009

Treutler, O., and Ahlrichs, R. (1995). Efficient molecular numerical integration schemes. J. Chem. Phys. 102, 346-354. doi: 10.1063/1.469408

von Arnim, M., and Ahlrichs, R. (1998). Performance of parallel Turbomole for density functional calculations. J. Comput. Chem. 19, 1746-1757. doi: $\quad$ 10.1002/(SICI)1096-987X(19981130)19:15<1746::AID-JCC7>3.0. $\mathrm{CO} ; 2-\mathrm{N}$

von Arnim, M., and Ahlrichs, R. (1999). Geometry optimization in generalized natural internal coordinates. J. Chem. Phys. 111, 9183-9190. doi: $10.1063 / 1.479510$

Weigend, F. (2006). Accurate Coulomb-fitting basis sets for H to Rn. Phys. Chem. Chem. Phys. 8, 1057-1065. doi: 10.1039/B515623H

Weigend, F., and Ahlrichs, R. (2005). Balanced basis sets of split valence, triple zeta valence and quadruple zeta valence quality for $\mathrm{H}$ to $\mathrm{Rn}$ : design and assessment of accuracy. Phys. Chem. Chem. Phys. 7, 3297-3305. doi: 10.1039/B508541A

Yousif, L. F., Stewart, K. M., and Kelley, S. O. (2009). Targeting mitochondria with organelle-specific compounds: strategies and applications. Chembiochem 10, 1939-1950. doi: 10.1002/cbic.200900185

Zhao, Y., and Truhlar, D. G. (2008). The M06 suite of density functionals for main group thermochemistry, thermochemical kinetics, noncovalent interactions, excited states, and transition elements: two new functionals and systematic testing of four M06-class functionals and 12 other functionals. Theor. Chem. Acc. 120, 215-241. doi: 10.1007/s00214-007-0310-x

Conflict of Interest: The authors declare that the research was conducted in the absence of any commercial or financial relationships that could be construed as a potential conflict of interest.

Copyright (c) 2020 Pala, Senn, Caldwell, Prime, Warrington, Bright, Prag, Wilson, Murphy and Hartley. This is an open-access article distributed under the terms of the Creative Commons Attribution License (CC BY). The use, distribution or reproduction in other forums is permitted, provided the original author(s) and the copyright owner(s) are credited and that the original publication in this journal is cited, in accordance with accepted academic practice. No use, distribution or reproduction is permitted which does not comply with these terms. 